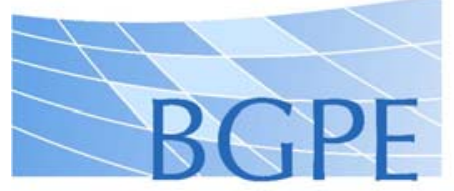

Bavarian Graduate Program in Economics

BGPE Discussion Paper

No. 143

\title{
The Multivariate Option iPoD Framework - Assessing Systemic Financial Risk
}

\author{
Philipp Matros \\ Johannes Vilsmeier
}

October 2013

ISSN 1863-5733

Editor: Prof. Regina T. Riphahn, Ph.D.

Friedrich-Alexander-University Erlangen-Nuremberg

(c) Philipp Matros, Johannes Vilsmeier 


\title{
The Multivariate Option iPoD Framework - Assessing Systemic Financial Risk ${ }^{1}$
}

\author{
Philipp Matros², Johannes Vilsmeier ${ }^{3}$
}

\begin{abstract}
We derive multivariate risk neutral asset distributions for major US financial institutions (FIs) using option implied marginal risk neutral asset distributions (RNDs) and probabilities of default (PoDs). The multivariate densities are estimated by combining the entropy approach, dynamic copulas and rank correlations. Our density estimates exhibit information about the conditional distributions of the individual FIs and we propose several financial distress measures based on default scenarios taking place in the financial sector. Empirical results around the period of the US sub-prime crisis show that the proposed risk measures in a timely manner identify i) the most troubled FIs in the system, ii) the systemically most important FIs, iii) the implicit bailout guarantees of some FIs and iv) a 'too interconnected to fail' problem in the US financial sector throughout the year 2008.

Keywords: Financial Distress, Conditional Probability of Default, Copulae, Option Prices, Entropy Principle

JEL classification: C14, C32, G01, G21
\end{abstract}

\footnotetext{
${ }^{1}$ We want to thank Rolf Tschernig and Enzo Weber for their valuable research assistance and the participants of the Econometric Research Seminars at the University of Regensburg and the Institut für Arbeitsmarkt und Berufsforschung (IAB), Nuremberg, as well as participants of the Research Workshops of the Bavarian Graduates Program in Economics (BGPE) for helpful comments. In addition, Johannes Vilsmeier wants to thank Giulia Iori for her assistance during his research visit at the City University, London and the BGPE for financial support during the research and writing process. Of course, all remaining errors are our own.

${ }^{2}$ Universität Regensburg, D-93040 Regensburg, Germany, philippmatros@yahoo.de

${ }^{3}$ Corresponding author. Universität Regensburg, D-93040 Regensburg, Germany, johannes.vilsmeier@gmx.de
} 


\section{Introduction}

Systemic financial risk deals with the risk of the financial sector as a whole as opposed to the risk of just individual financial entities. In order to measure this type of risk it is necessary to assess beside the risk of individual financial institutes (FIs) also the risk that arises due to the interconnectedness among the FIs in the economy. Interconnectedness in the financial sector occurs due to direct links, as e.g. through the interbank market, and/or indirect links arising e.g. from similar exposures in the FIs portfolios. These linkages can cause that financial distress in one or a small group of FIs spills over to other firms (contagion) and propagates within the financial sector to a large systemic crisis, as e.g. seen during the 2007/2008 sub-prime crisis. Assessments of the sub-prime crisis at the G20 summits in Washington 2008 and subsequent summits resulted in an action plan to implement a so called macro-prudential policy framework whose objective is to mitigate systemic risks and reduce the likelihood of future financial crises (Jenkins and Thiessen (2012); BIS (2011)). Opposed to traditional, micro-prudential regulation, that focuses on the soundness of single FIs, macro-prudential policy focuses on the risk of the financial system as a whole. This is due to the recognition that the interconnectedness of FIs and markets increases financial risk to an extent that is not captured by focusing solely on individual institutions (Group of Thirty (2010), Bernanke (2010)). As part of the macro-prudential agenda, in recent years, institutions such as the European Systemic Risk Board (ERSB) or the Financial Stability Oversight Council (FSOC) in the US were founded to assess systemic risk and to identify systemic important FIs (SIFIs). Further, new regulatory frameworks as Basel III (global) and the Frank-Dodd Act (USA) implemented macro-prudential policy instruments such as anti-cyclical capital requirements and additional regulatory requirements for SIFIs (Murphy (2013), BoE (2009)). A prerequisite for successful macro-prudential policy is the timely measurement of systemic risk as well as the identification of the most vulnerable and the systemically most important FIs.

In this paper we suggest a novel framework to derive informative measures for systemic financial risk. Our approach combines the (univariate) option iPoD framework, proposed in Capuano (2008) and Vilsmeier (2011), and the most entropic copula procedure, proposed in Chu (2011), such that we can estimate multivariate asset distributions (MADs) for a sample of financial institutions (FIs). The approach, that we refer to as the multivariate option iPoD procedure, derives the MADs in such a way that they exhibit information about the conditional Probabilities of Default (PoDs) of the FIs in the sample. Hence, we are able to implement distress scenarios that are based on the default of one or several institutions in the system and evaluate the impact on the residual FIs in the system. The distress levels 
measured by the proposed risk indicators incorporate the firm's individual risk level as well as risk induced by distress in other firms of the system and spilt over due to the dependence among the FIs.

The cornerstone of our methodology is the option iPoD approach, in which option implied risk neutral densities (RNDs) are estimated in such a way that they reveal information about the PoD of the issuer of the underlying. Default in this framework corresponds to the event that the stock price of the firm falls to zero during the time to maturity of the options. Our framework to derive firm's PoDs is purely statistical and does neither require balance sheet nor recovery rate information in contrast to competing methods like Merton-type models ${ }^{4}$ and Reduced Form (RF) models ${ }^{5}$. The RNDs are estimated using a semi-parametric estimation procedure based on the entropy concept (Shannon (1948)) and moment constraints that are given by risk neutral pricing theory. As illustrated in Vilsmeier (2011) the estimated distributions are in general very smooth and highly flexible with regard to their functional forms such that the empirically observed deviations of RNDs from normality can be easily modeled. Further, the framework provides highly plausible PoD estimates, which, as shown in Matros and Vilsmeier (2012), clearly outperform CDS spreads in identifying the most vulnerable FIs in the course of 2007/2008. Following the suggestions in Matros and Vilsmeier (2012) we derive daily time series of maturity corrected RNDs, where each RND is estimated using only daily available option prices for different strikes and a given maturity.

To extend the option iPoD procedure to a multivariate framework we apply the statistical concept of copulas. Copulas are a very flexible way to estimate multivariate probability distributions, as they allow to model the dependence structure of a multivariate distribution (the copula) independently of its univariate marginals. Based on Sklar's theorem (Sklar (1959)) we can combine a given copula with arbitrary marginals and obtain different multivariate distributions. The most entropic copula (MEC) approach of Chu (2011) allows to model the dependence structure between the different FIs on basis of Spearman rank correlations that we estimate on basis of the time series of marginal RNDs. We estimate the rank correlations dynamically with exponentially decreasing influence of past observations and

\footnotetext{
${ }^{4}$ Merton-type models define a firm's default according to the structural approach of Merton (1974), which states that a firm defaults if its value of assets is lower than its value of debt. To calculate the PoD one calibrates asset distributions on basis of historical equity prices and defines a default point according to the firm's book value of debt. Examples of structural approaches to measure systemic risk are: Tudela and Young (2005), Chan-Lau and Gravelle (2005), Lehar (2005), Crosbie and Kocagil (2003), J.P.Morgan (1997).

${ }^{5}$ Reduced form models use debt based market instruments and their market prices to calibrate the firms' default processes (usually modelled as Poisson processes). To calculate the implied PoDs, assumptions about unknown recovery rates in the case of the firm's default have to be made. Examples for reduced form models applied to measuring systemic risk are: Huang et al. (2012), Segoviano and Goodhart (2009), Avesani et al. (2006).)
} 
obtain time-varying measures of dependence that can capture linear as well as non-linear dependence and may change throughout the economic cycle. By applying Sklar's theorem we combine the RNDs from the (univariate) option iPoD approach and the MEC to obtain time series of MADs whose marginals and dependence structure may change every day. As the framework is easily implemented in higher dimensions (i.e. $d>2$ ) our methodology provides a high-dimensional, dynamic, non-Gaussian Copula framework that is mathematically tractable and straightforward to implement. This clearly contrasts our methodology to standard approaches of Merton-type and RF models that usually model the dependence structures between different firms in a static and linear way, based on historical equity return Pearson correlations.

We apply our framework to data of 13 of the largest US FIs during the period of January 2007 to September 2008 and calculate time series of five different distress indicators. The indicators are derived on basis of conditional PoDs (CPoDs) and conditional lower quantiles (CQs) of the MAD. Compared to CPoDs, the CQ measures can be interpreted as broader definitions of distress that cover beside default also events like downgrades or large losses in market value. To our knowledge, these CQ risk measures are newly introduced to the literature. The estimated distress indicators are in a timely manner able to identify i) the most troubled FIs during $2007 / 2008$, i) the systemically most important FIs, iii) the implicit bailout guarantees conceded to some FIs and iv) the high degree of interconnectedness in the sector throughout the year 2008.

The contributions of our paper are as follows. We introduce a novel framework to measure systemic financial risk by estimating a financial sector's multivariate asset distribution (MAD). This framework has several attractive properties: i) the MADs incorporate information about the firm's PoDs and conditional PoDs ii) we do neither require balance sheet nor recovery rate information do derive the MADs, iii) the estimated MADs are high-dimensional, capture dynamic and non-linear dependence structures, and are highly flexible with regard to their functional forms. Based on the MADs that we estimated for 13 major US FIs during the period of January 2007 to September 2008, we calculate five different distress indicators based on conditional PoDs and conditional lower quantiles. The analysis of the distress indicators shows the high informational content of the framework which provides very plausible results given the historical events during the US sub-prime crisis.

The remainder of the paper is structured as follows. First, section 2 introduces the methodology of the multivariate option iPoD procedure. Section 3 presents the data used in our empirical application to the US financial sector. In section 4, we give a brief description on how to empirically implement the framework, and section 5 provides the estimation results. 
Finally, section 6 concludes and gives some suggestions for future research.

\section{Methodology}

The multivariate option $\mathrm{iPoD}$ methodology uses copula theory to estimate MADs for a sample of FIs. Using this theory one can model the univariate asset distributions (RNDs) of the FIs independently of their dependence structure (copula) and combine them to MADs using Sklar's theorem (Sklar (1959)). We apply the univariate option iPoD approach as suggested in Vilsmeier (2011)) to estimate the univariate asset distributions, and the most entropic copula (MEC) approach of Chu (2011) to model the dependence structure. Both frameworks apply a semi-parametric estimation procedure which maximizes the entropy function (Shannon (1948)) under moment constraints. In the case of the option iPoD procedure the moment constraints are obtained from the theory of risk neutral pricing and the observed option prices for different strikes. In the case of the MEC the moment constraints are given by the dynamic Spearman rank correlations that are estimated on basis of the marginal RND time series. In the following we describe the individual building blocks of our framework in detail.

\section{Copulas}

A copula $C$ can be isolated from any multivariate random vector $\left(X_{1}, \ldots, X_{n}\right)$ with multivariate distribution function $H\left(x_{1}, \ldots, x_{n}\right)=P\left(X_{1} \leq x_{1}, \ldots, X_{n} \leq x_{n}\right)$ and marginal distributions $F_{i}\left(x_{i}\right)=P\left(X_{i} \leq x_{i}\right), i=1, \ldots, n$, by transforming the univariate random variables $X_{i}$ to standard-uniform random variables using the probability integral transform, i.e. by applying the marginal cdfs $F_{i}$ onto each random variable $X_{i}$ leading to $U_{i}=F_{i}\left(X_{i}\right) \sim U(0,1)$. The distribution function of the resulting random vector $\left(U_{1}, \ldots, U_{n}\right)$ is given by the copula function, i.e.:

$$
C\left(u_{1}, \ldots, u_{n}\right)=P\left(U_{1} \leq u_{1}, \ldots, U_{n} \leq u_{n}\right)=H\left(X_{i} \leq F_{x_{i}}^{-1}\left(u_{i}\right), \ldots, X_{n} \leq F_{x_{n}}^{-1}\left(u_{n}\right)\right) .
$$

This means that a copula is a multivariate distribution function $C:[0,1]^{d} \rightarrow[0,1]$ with standard uniform (univariate) marginals. ${ }^{6}$

According to Sklar's theorem (Sklar (1959)) there exists a copula for all $H$ and $F_{i}, \ldots, F_{n}$ such

\footnotetext{
${ }^{6}$ Formally a copula function satisfies the following three properties:

$$
\begin{gathered}
C\left(1, \ldots, 1, u_{i}, 1, \ldots, 1\right)=u_{i} \quad \text { for every } i \leq n \text { and all } u_{i} \text { in }[0,1] . \\
C\left(u_{1}, \ldots, u_{n}\right)=0 \quad \text { if } u_{i}=0 \text { for any } i \leq n .
\end{gathered}
$$
}

$C$ is $n$-increasing, i.e. for each $n$-box $R:=\prod_{i=1}^{n}\left[x_{i}, y_{i}\right] \subseteq[0,1]^{n}, x_{i} \leq y_{i}$, the $C$-volume $V_{C}(R)$ is non-decreasing: 
that for all $\left(x_{1}, \ldots, x_{n}\right) \in \overline{\mathbb{R}}^{n}$ :

$$
H\left(x_{1}, \ldots, x_{n}\right)=C\left(F_{1}\left(x_{1}\right), \ldots, F_{d}\left(x_{n}\right)\right),
$$

where $C$ is unique if all $F_{i}$ are continuous. Using Sklar's theorem we can create for a given copula different $H$, which all exhibit the same dependence structure but differ by their marginals $F_{i}$. Conveniently, the different marginals may come from different families of probability distributions. As copulas allow to model the dependence structure and the marginals independently from each other and subsequently combine them arbitrarily, they provide a much more flexible framework to model multivariate densities than fitting parametrized multivariate distributions to the data. ${ }^{7}$

An other important property of copulas is that they are a 'non-parametric' measure of dependence since they are invariant under strictly increasing and continuous transformations of their marginals. In contrast to traditional linear correlation coefficients, rank correlation measures share this property since applying the probability integral transform to a random variable corresponds to a rank transformation of that random variable. This implies a direct representation of rank measures of dependence as a function of the copula. The Spearman rank coefficient that will be used in the context of the MEC estimation has the following copula representation (see e.g. Nelsen (2006), chapter 5):

$$
\rho\left(X_{i}, X_{j}\right)=12 \int_{0}\left(C\left(u_{i}, u_{j}\right)-u_{i} u_{j}\right) d u_{i} d u_{j}-3
$$

\section{Most Entropic Copula (MEC) Approach}

In order to estimate the MAD we need to find a copula that adequately captures the dependence structure among the different RNDs. Standard approaches assume specific parametric copulas, such as the Gaussian- or t-copula, and estimate the parameters such that they fit the data at hand. Since different parametric assumptions impose different dependence structures onto the data, the results in empirical applications can be quite sensitive to the specific choice of the copula, as shown in Frey et al. (2001). The MEC approach of Chu (2011) circumvents the copula choice problem too a large degree by estimating a semi-parametric copula that,

$V_{C}(R):=\sum \operatorname{sgn}(\mathbf{z}) C(\mathbf{z}) \geq 0$, where the sum is over all vertices $\mathbf{z}$ of $\mathrm{R}$ and $\operatorname{sgn}(\mathbf{z})=\left\{\begin{aligned} 1 & z_{k}=a_{k} \text { for even } k \mathrm{~s} \\ -1 & z_{k}=a_{k} \text { for odd } k \mathrm{~s}\end{aligned}\right.$

${ }^{7}$ For a in depth description of the copula concept see e.g. Nelsen (2006). 
from an information theoretic point of view, imposes as little information as possible on the shape of the copula beyond what is actually known from the data. This is achieved by applying the entropy principle to identify the optimal copula. The entropy principle, formulated by Jaynes (1957), states that given the information from the data (expressed as moment constraints) the distribution which best describes the current state of knowledge is the one that maximizes the entropy function.

The entropy function for a multivariate density function $f\left(x_{1}, \ldots, x_{n}\right)$ is defined as:

$$
H\left[f\left(x_{1}, \ldots, x_{n}\right)\right]=-\int_{-\infty}^{\infty} f\left(x_{1}, \ldots, x_{n}\right) \log f\left(x_{1}, \ldots, x_{n}\right) d x_{1} \ldots x_{n} .
$$

As shown by Shannon (1948), the entropy function can be be interpreted as a metric measure for the average uncertainty in a random variable, where the uncertainty refers to the predictability of an (average) outcome of the random variable. Jaynes (1957) suggested to use the entropy function for density estimation purposes when there is information about the density in form of expected values available (moment constraints).

In the MEC approach a copula density function $c\left(u_{1}, \ldots, u_{n}\right)$ is estimated by maximizing the entropy function subject to moment constraints that are given by i) constraints that guarantee that the $n$ marginals of the density are uniform and ii) rank correlation functions expressed in terms of $c\left(u_{1}, \ldots, u_{n}\right)$ that have to satisfy their empirical counterparts estimated from the data.

The MEC framework is very flexible with regard to modeling different types of dependence. As suggested in Chu (2011) different kinds of rank measures can be jointly used in the estimation. On the one hand, these can be traditional rank measures as Spearman's $\rho$ or Kendall's $\tau$, which are non-parametric measures and capture linear and non-linear dependence structures. On the other hand, one can use measures like Blest measures of correlation (Blest (2000)) or conditional Spearman coefficients (Schmid and Schmidt (2007)), which allow to model asymmetric types of dependence or specific types of tail-dependence. ${ }^{8}$ On the downside, as for the estimation of a $n$-dimensional copula all possible pairwise rank correlations among the different RNDs have to be included, each type of rank measure (e.g. Spearman's $\rho)$ implies $n(n+1) / 2$ additional constraints and hence additional parameters to estimate. We will restrict ourselves to a set-up including solely ordinary Spearman rank correlations but which will be estimated in a dynamic way such that possible asymmetric dependence structures should be captured and displayed too a large extent in the time-varying correla-

\footnotetext{
${ }^{8}$ Another possible and interesting approach is to include multivariate versions of Spearman's $\rho$, or of other rank measures. Examples of multivariate rank measures are e.g. given in Schmid and Schmidt (2007).
} 
tions.

\section{The MEC Estimation Setup}

The above described estimation problem is formalized in a $n$-dimensional set-up as follows:

$$
\text { Maximizing } H\left[c\left(u_{1}, \ldots, u_{n}\right)\right]=-\int_{[0,1]^{n}} c\left(u_{1}, \ldots, u_{n}\right) \log c\left(u_{1}, \ldots, u_{n}\right) d u_{1} \ldots d u_{n}
$$

subject to:

$$
\begin{gathered}
\int c\left(u_{1}, \ldots, u_{n}\right) d u_{1} \ldots d u_{n}=1 \\
\int_{[0,1]^{n}} \int_{\left[0, u_{i}\right][0,1]^{n-1}} u_{i}^{j} c\left(u_{1}, \ldots, u_{i}, \ldots, u_{n}\right) d u_{1} \ldots d u_{i} \ldots d u_{n}=\frac{1}{1+j}
\end{gathered}
$$

$\forall i=1,2, \ldots, n$ and $j=1,2, \ldots, m$,

$$
\int_{[0,1]^{n}} u_{k} u_{l} c\left(u_{1}, \ldots, u_{n}\right) d u_{1} \ldots d u_{n}=\frac{\widehat{\rho}^{k, l}+3}{12}
$$

$\forall k=2,3, \ldots, n, l=1,2, \ldots, n-1, l<k$.

Equation (6) is the entropy function with the multivariate copula density $c\left(u_{1}, \ldots, u_{n}\right)$ as its argument. Equation (7) represents the additivity constraint which guarantees that the copula density integrates up to one. The set of equations (8) guarantees that the $n$ marginals of the copula are standard-uniform by imposing that the first $m$ moments (with $m$ as finite integer) of the copula density satisfy the theoretical first $m$ moments of a standard-uniform, where the $j$-th moment is given by $\mu_{j}=1 /(1+j)$. This is of course only an approximation but as shown in Chu (2011) already a relatively small set of constraints guarantees approximate standard-uniform marginals.

The system of equations (9) represents the dependence constraints given by the estimated Spearman's $\rho$ in terms of the copula density and the pairwise Spearman rank correlations among all $n$ FIs estimated from the respective RNDs. Given the RND time series for the different FIs, we obtain the empirical correlations by drawing for each firm randomly from its RNDs at all points in time such that we get a time series for the respective firm's asset value. On basis of each pair of time series of different firms we can estimate the rank correlation. This process is carried out repeatedly such that we obtain a distribution of Spearman's $\rho$ for each pair of firms, and we take the median from this distribution as our estimate $\widehat{\rho}^{k, l}$. To model a time-varying dependence structure between the FIs, we estimate the correlation coefficients dynamically with exponentially decreasing influence of past observations. Following 
the suggestions in RiskMetricsTM (1996) our empirical correlation estimates are obtained according to:

$$
\rho_{t}^{k, l}=\frac{\sum_{s=1}^{t-1} \alpha^{t-s-1}\left(R_{k, s}-\bar{R}_{k}\right)\left(R_{l, s}-\bar{R}_{l}\right)}{\sqrt{\sum_{s=1}^{t-1} \alpha^{t-s-1}\left(R_{k, s}-\bar{R}_{k}\right)^{2}\left(R_{l, s}-\bar{R}_{l}\right)^{2}}},
$$

where $\alpha^{t-s-1}$ denotes the weighting coefficient, with $\left.\alpha \in\right] 0,1[$ and $t$ as a moving endpoint that is equal to the period for which we calculate the respective correlation coefficient ('stretching window' approach). ${ }^{9} R_{k, s}$ and $R_{l, s}$ are the rank transformed asset values in time period $s$ for two different firms, and $\bar{R}_{k}=\frac{1}{t-1} \sum_{s=1}^{t-1} R_{k}, s$ and $\bar{R}_{l}=\frac{1}{t-1} \sum_{s=1}^{t-1} R_{l}, s$ are the respective means of the rank transformed data. Equation (10) provides us with daily updated rank correlations and we obtain a framework in which the dependence structure among the FIs may change every day (dynamic copula approach).

The estimation of the copula function represented by the maximization set-up (6)-(9) can be carried out using ordinary Lagrange multiplier methods. Deriving the Lagrangian with respect to $c\left(u_{1}, \ldots, u_{n}\right)$ yields for a given time period $t$ the following form for the optimal density:

$$
c_{t}^{*}\left(u_{1} \ldots u_{n}\right)=\exp \left\{-\sum_{i=1}^{n} \sum_{j=1}^{m} \lambda_{i, j} u_{i}^{j}-\sum_{\substack{k=2 \\ l=1 \\ l<k}}^{n-1} \lambda_{n+k-1, l} u_{k} u_{l}\right\}
$$

where the set of $\lambda \mathrm{s}$ (the Lagrange multipliers) is obtained from the minimum of the following function (for a derivation see e.g. Chu (2011) or Alhassid et al. (1978)):

$$
Q(\lambda)=\int_{[0,1]^{n}} \exp \left\{-\sum_{i=1}^{n} \sum_{j=1}^{m} \lambda_{i, j}\left(u_{i}^{j}-\frac{1}{1+j}\right)-\sum_{k=2}^{n} \sum_{\substack{l=1 \\ l<k}}^{n-1} \lambda_{n+k-1, l}\left(u_{k} u_{l}-\frac{\widehat{\rho}_{t}^{k, l}+3}{12}\right)\right\} d u_{1} \ldots d u_{n} .
$$

\section{The option iPoD framework}

Cornerstone of our MAD estimation approach is the (univariate) option iPoD framework which allows us to estimate the required time series of univariate asset distributions (RNDs) for all FIs considered in our sample. While the theoretical foundations of the option iPoD methodology were introduced by Capuano (2008) and Vilsmeier (2011), Matros and Vilsmeier (2012) show how the framework can be empirically implemented in such a way that time

\footnotetext{
${ }^{9}$ Of course there are some theoretically more appealing but also more complex methodologies to derive the rank correlations in a dynamic way. Especially the use of realized correlations theory (e.g. BarndorffNielsen and Shephard (2004)) might be a promising way to make the correlations less dependent on historical data.
} 
series of maturity corrected RNDs/PoDs can be derived. We closely follow the suggestions made in Matros and Vilsmeier (2012).

The option iPoD procedure allows to estimate risk neutral densities in such a way that they exhibit information about the default probability of the issuing company of the option's underlying. Default of a firm is triggered when the stock price $S$ of a firm falls to zero during time to maturity of the option. Importantly, the PoDs and RNDs are derived in a purely statistical manner without the use of balance-sheet information or recovery rate assumptions. Solely up-to-date information given by the set of daily observable equity (call) option prices to different strike prices $K_{i}$ (and by a risk-free interest rate) is required for the estimation.

The idea of the option iPoD framework is to modify traditional RND estimation approaches such that it is possible to estimate a 'mass point' in the RND that indicates the probability that the underlying of a stock option will have value zero at time of maturity $T$. The mass point is given as the integral over the density in a specific sub-domain of the RND. This subdomain is obtained by shifting the domain of possible realizations for $S_{T}$ upwards by some constant $D$, and to define a new variable $V_{T}=S_{T}+D$. Then the RND $f\left(V_{T}\right)$ is estimated for this new variable. Using the domain of the new variable the pay-off for a call option in $T$ with strike $K_{i}$ is defined by: $C_{T}^{K_{i}}=\max \left(V_{T}-D-K_{i} ; 0\right)$. Hence, there will be no pay-off for the option in the interval of values $V_{T} \in[0, D]$. The applied estimation procedure ensures that there is an interaction between the (entire) density of the RND for $V_{T} \in\left[D, V_{\text {max }}\right]$, with $V_{\max }$ as upper domain bound for $V_{T}$, and the level of the PoD (i.e. the size of the density assigned to $\left.V_{T} \in[0, D]\right)$ such that the combination of the RND shape and the PoD level that best fits the observed prices can be identified (see Vilsmeier (2011)). The choice of $D$ is crucial. It determines the length of the sub-domain $[0, D]$ and jointly with it the PoD. Using numerical experiments, Vilsmeier (2011) found that for arbitrary reasonable RND forms and PoD levels, the procedure can approximate the respective probability mass point for $S_{T}=0$ quite well if the constant $D$ is chosen within the interval $[1 ; 20]$. Since an exact rule for the determination of the optimal $D$ has not yet been detected, the PoDs are obtained by averaging over RND estimates with different $D$ s ranging from 1 to 20 . The optimal RND is then identified as the one that provides the PoD closest to the average PoD ('averaging approach'). Matros and Vilsmeier (2012) found that the 'averaging approach' provides highly plausible and informative estimates in various empirical applications.

Despite the purely statistical nature of the option iPoD approach, one may give the variables a theoretical meaning by applying a Merton (1974) type interpretation. In this case $V_{T}$ denotes the value of assets, $S_{T}$ represents the value of equity and $D$ denotes the value of debt. Using this interpretation, a firm defaults if the value of assets does not cover the value of debt. 
In order to obtain the risk-neutral density $f\left(V_{T}\right)$ for a given $D$, we use moment constraints given by the continuous risk neutral option pricing formula (Cox and Ross (1976)) and the observed option prices for different strikes $K_{i}$ at time $t=0$ :

$$
C_{0}^{K_{i}}=e^{-r T} \int_{V_{T}=D+K_{i}}^{V_{\max }}\left(V_{T}-D-K_{i}\right) f\left(V_{T}\right) d V_{T}, \quad i=1, \ldots, B .
$$

Equation (13) depends on the unknown RND $f\left(V_{T}\right)$ for observable option prices $C_{0}^{K_{i}}$ at different strikes. The formula states that the today's observed option prices must be equal to the discounted expectation over all possible pay-offs under the risk neutral probability density, where $B$ denotes the number of observable option prices for different strikes $K^{10}$ and $r$ represents the annualized risk free rate. The current stock price $S_{0}$ is included as an option with strike $K_{1}=0$. One faces an under-determined estimation problem as there is an infinite number of densities compatible with a finite number of moment conditions. In order to obtain an unique solution for $f\left(V_{T}\right)$ the principle of maximum entropy is used. In contrast to the MEC estimation set-up from above, the option iPoD approach uses the related cross-entropy (CE) function to derive the optimal density and not the entropy function. The CE function, introduced by Kullback and Leibler (1951)), minimizes the so-called entropic distance (see e.g. Cover and Thomas (2006), chapter 2), $C E(x)=\int_{0}^{V_{\max }} f(x) \log \frac{f(x))}{f^{0}(x)}$, between the density of interest $f(x)$ to be determined and some prior function $f^{0}(x)$. The CE function allows for a more general density estimation set-up than the entropy function by providing the possibility to consider a priori information in the estimation process. Importantly, in the case of a finite support and uniform prior, the minimization of the CE function and the maximization of the entropy function lead to the same optimal density for any given set of moment constraints. 11

Given the moment constraints in (13) and an additivity constraint $\int_{0}^{V_{\max }} f\left(V_{T}\right) d V_{T}=1$, which ensures that the density integrates up to one, the RND estimation problem of finding $f\left(V_{T}\right)$

\footnotetext{
${ }^{10}$ Note that the strike prices in equation (13) are denoted in increasing order, i.e. $\mathrm{i}=1$ denotes the smallest and $\mathrm{i}=\mathrm{B}$ the largest strike price.

${ }^{11}$ More precisely, the prior function has to be of maximal entropy on the defined domain for $x$. On a closed interval this will be the uniform distribution, on an unbounded positive real valued domain (for a given mean) the exponential distribution and on a unbounded real valued interval (given a mean and a variance) the normal distribution.
} 
can be formalized by the following Lagrangian function:

$$
\begin{array}{r}
L=\int_{V_{T}=0}^{V_{\max }} f\left(V_{T}\right)\left[\log \frac{f\left(V_{T}\right)}{f^{0}\left(V_{T}\right)} d V_{T}\right]+\lambda_{0}\left[1-\int_{V_{T}=0}^{V_{\max }} f\left(V_{T}\right) d V_{T}\right] \\
+\sum_{i=1}^{B} \lambda_{i}\left[C_{0}^{K_{i}}-e^{-r T} \int_{V_{T}=D+K_{i}}^{V_{\max }}\left(V_{T}-D-K_{i}\right) f\left(V_{T}\right) d V_{T}\right]
\end{array}
$$

where $f^{0}\left(V_{T}\right)$ denotes a uniform prior function on the interval $\left[0, V_{\max }\right]$.

Optimizing (14) with respect to $f\left(V_{T}\right)$ yields (see e.g. Cover and Thomas (2006), chapter 12):

$$
f^{*}\left(V_{T}\right)=\frac{1}{\mu(\lambda)} f^{0}\left(V_{T}\right) \exp \left[\sum_{i=1}^{B} \lambda_{i} e^{-r T} \mathbf{1}_{V_{T}>D+K_{i}}\left(V_{T}-D-K_{i}\right)\right]
$$

with

$$
\mu(\lambda)=\exp \left(1-\lambda_{0}\right)=\exp \left(-\lambda_{0}^{\prime}\right)=\int_{V_{T}=0}^{V_{\max }} f^{0}\left(V_{T}\right) \exp \left[\sum_{i=1}^{B} \lambda_{i} e^{-r T} \mathbf{1}_{V_{T}>D+K_{i}}\left(V_{T}-D-K_{i}\right)\right] d V_{T}
$$

Under the assumption of a finite domain for $V_{T} \in\left[0, V_{\max }\right]$, the optimal set of Lagrange multipliers $\lambda_{i}, i=1,2 \ldots B$, is obtained as the minimum of the following function (see Vilsmeier (2011) for a derivation):

$$
\begin{aligned}
& F=\log \left(\frac{1}{V_{\max }}\right)+\log \left\{\exp \left(-\sum_{i=1}^{B} w_{i} \lambda_{i} C_{0}^{K_{i}}\right) D\right. \\
& -\sum_{i=1}^{B-1}\left[\frac{\exp \left(\sum_{j=1}^{i} w_{j} \lambda_{j}\left(e^{-r T}\left(K_{i}-K_{j}\right)-C_{0}^{K_{j}}\right)-\sum_{k=i+1}^{B} w_{k} \lambda_{k} C_{0}^{K_{k}}\right)}{e^{-r T}\left(\sum_{j=1}^{i} w_{j} \lambda_{j}\right)}\right. \\
& \left.-\frac{\exp \left(\sum_{j=1}^{i} w_{j} \lambda_{j}\left(e^{-r T}\left(K_{i+1}-K_{j}\right)-C_{0}^{K_{j}}\right)-\sum_{k=i+1}^{B} w_{k} \lambda_{k} C_{0}^{K_{k}}\right)}{e^{-r T}\left(\sum_{j=1}^{i} w_{j} \lambda_{j}\right)}\right] \\
& \left.-\left[\frac{\exp \left(\sum_{j=1}^{B} w_{j} \lambda_{j}\left(e^{-r T}\left(K_{B}-K_{j}\right)-C_{0}^{K_{j}}\right)-\exp \left(\sum_{j=1}^{B} w_{j} \lambda_{j}\left(e^{-r T}\left(V_{\max }-D-K_{j}\right)-C_{0}^{K_{j}}\right)\right.\right.}{e^{-r T}\left(\sum_{j=1}^{B} w_{j} \lambda_{j}\right)}\right]\right\}
\end{aligned}
$$

where $w_{i}$ denote liquidity weights that are pre-multiplied to the Lagrange multipliers $\lambda_{i}$. The 
weights ensure that more liquid option contracts (measured in our approach in terms of open interest) have to be approximated more closely by the estimated RND. As stressed in Matros and Vilsmeier (2012), the assignment of the liquidity weights is very important in order to obtain timely consistent and smooth PoD estimates.

The option iPoD framework has several appealing properties that are of interest when assessing systemic financial risk. First, as the methodology provides PoD estimates, it allows to implement default scenarios in the financial sector and to assess (in combination with the MEC approach) the impact onto the resilience of the financial system. Second, the PoDs are derived without the use of balance-sheet data or recovery rate assumptions. As pointed out e.g. in Vilsmeier (2011), the assumptions made in Merton-type models and RF approaches are especially severe in applications to FIs. This is due to the fact that these type of firms exhibit complex, opaque and very volatile asset and liability structures. This makes the exact definition of default points, as required by Merton-type models, or the estimation of recovery rates, as necessary for RF frameworks, very difficult. Third, compared to recently suggested frameworks such as Segoviano and Goodhart (2009), that exclusively use PoD estimates to model a financial sector's MAD, our approach offers the possibility to derive beside CPoD estimates also broader measures of distress based on the CQs of the MADs. This is due to the fact that the PoDs are estimated jointly with the corresponding RNDs. As we will see in section 5, the CQ measures make it possible to identify governmental bailout guarantees conceded to some FIs.

\section{Data}

To calculate the MAD for a set of firms on a specific day, one requires a risk free interest rate and for each firm the prices of the call options written on this firm's stock to different strike prices (including the stock price itself). As the risk free interest rate we use the 3-month treasury bill secondary market rate obtained from the FRED data base. The option data are daily option closing prices for contracts with maturities ranging from five to seven months which were obtained from the New York Stock Exchange (NYSE) via the data provider Stricknet.

Our option data set comprises call prices for 13 different FIs ranging from January 1, 2003 to September 10, 2008. While we use the entire sample to calculate the RNDs and the dynamic Spearman correlations, we calculate the MADs from January 1, 2007 to September 10, 2008. The considered institutions in our sample are: Goldman Sachs (GS), Wells Fargo (WFC), Citigroup (C), Bank of America (BAC), JPMorgan Chase (JPM), the American 
International Group (AIG), Morgan Stanley (MS), Lehman Brothers (LEH), Bear Stearns (BSC), Wachovia Bank (WB), Merill Lynch (MER), Countrywide Financial (CFC) and Washington Mutual (WM). All of these institutions were among the largest US FIs during the period of $2007 / 2008$.

The time span for which we calculate the MADs covers the beginning sub-prime crisis period with growing losses in the financial sector and first (smaller) collapses in 2007 until shortly before the big burst of the crisis around September 15, 2008 which in the following led to a nearby collapse of the US financial system. Our sample contains several FIs involved in incisive events of the sub-prime crisis. These are: the rescue/takeover of BSC at March 15, 2008 by JPM (orchestrated and financially backed by the US Federal Reserve Bank (FED)); the takeover of CFC on July 1, 2007 by BAC; the collapse of LEH on September 15, 2008; the takeover of MER by BAC on September 14, 2008; the rescue of AIG by the FED at September 16, 2008; the collapse of WM on September 26, 2008; the announcement of the takeover of WB by WFC on October 3, 2008 (the purchase was finalized on December 31, 2008); and the rescue of Citigroup by the FED on November 23, 2008 (see e.g. Wheelock (2010)). Given this historical time line we make the following classifications for the FIs in our sample:

1. 'Surviving' FIs: JPM, GS, WFC, BAC, MS

2. Acquired FIs: BSC, CFC, WB, MER

3. 'Rescued' FIs: BSC, AIG, C, WB

4. 'Bankrupt' FIs: LEH, WM

The historical classification gives us a rough guideline about how to assess the informational content of our risk measures. In general we will assume that the 'surviving' FIs were less troubled during the crisis than the other institutions. In contrast, one can expect the 'bankrupt' banks to be highly troubled and, hence, should be indicated as very risky. For the 'acquired/rescued' FIs there might be effects of implicit government or bailout guarantees in our estimated MADs as such guarantees, if anticipated by the market, would influence the risk perception of the investors for these institutes.

\section{Empirical Implementation}

As described in section 2, the first step to implement the multivariate option iPoD framework is to estimate univariate RNDs for all FIs in our sample. This is done using the univariate option iPoD approach as described in Matros and Vilsmeier (2012). Following their sugges- 
tions, we first calculate the RNDs on basis of an option maturity cycle of 5-, 6-, and 7 months, and use open interest ${ }^{12}$ for the contracts as liquidity weights in the optimization of equation (17). The described maturity cycle means that we use a newly initiated 7-month contract for a specific firm for two months, until a new 7-months contract is initiated. For the RND domain we define a finite interval $\left[0, V_{\max }\right]$ for $V_{T}$, where $V_{\max }$ is set equal to five times the current stock value. To remove the maturity dependence inherent to the estimates, we apply the regression based maturity dependence correction scheme to the estimates described in Matros and Vilsmeier (2012). However, instead of using it solely for the PoDs we also apply it to the first ten moments of the RND estimates. In the correction scheme, the maturity effect on the RNDs is calculated by carrying out a (pooled) non-linear quantile regression of the RND estimates onto the respective time to maturity of the option contracts used to estimate the RNDs. We obtain correction factors and apply these to the moments and PoDs. To obtain a maturity corrected RND we use the cross-entropy procedure with a uniform prior and estimate a density that satisfies the moment constraints given by the maturity corrected moments and PoDs. ${ }^{13}$ Carrying out this procedure for every day, we eventually obtain a daily time series of maturity corrected RNDs for each of the 13 considered FIs for the period from January 1, 2003 to September 10, 2008. After the maturity correction, the RNDs imply a theoretical time to maturity and, hence, evaluation/forecast horizon of 7 months. ${ }^{14}$

In the second step we use the time series of the RNDs to estimate all possible dynamic Spearman rank correlations among the 13 RND time series (in total 78). To do so we sample for each FI randomly from its RNDs in order to obtain time series of their asset values $V_{T}$. Then we calculate the Spearman correlation coefficients dynamically and with exponentially decreasing influence of past observations between the time series. As persistence parameter in the exponential weighting scheme (see equation (9)) we use $\alpha=0.99$ as we assume that the dependence among the FIs is subject to some inertia. ${ }^{15}$ We repeat this procedure 5000 times such that we obtain a distribution of Spearman coefficients for each pair of FIs. Finally, we use the median from these distributions as our empirical estimate of the Spearman correlations

\footnotetext{
${ }^{12}$ The use of open interest (contracts traded in the past and not exercised or evened up yet) results in less volatile RND/PoD estimates than the use of trading volume. The weights are calculated by dividing open interest for a specific strike by the sum of open interest over all available strikes for a firm's stock option.

${ }^{13}$ The Lagrangian of the estimation set-up for a specific firm and time period $t$ is equal to equation (14) where the third term is replaced by: $\sum_{i=1}^{10} \lambda_{i}\left(M_{i}-\int_{0}^{V_{\max }}\left(V_{T}-\overline{V_{T}}\right)^{i} f\left(V_{T}\right) d V_{T}\right)$, with $M_{i}$ as the $i$-th central moment, and $\lambda_{11}\left(P o D-\int_{0}^{D} f\left(V_{T}\right)\right)$.

${ }^{14}$ In fact, the informational content in the used option prices implies an average time to maturity of 6 months as this is the average time to maturity of the used option contracts.

${ }^{15}$ Note, the weight of a one year (=250 days) old observation is $\approx 8 \%$ with $\alpha=0.99, \approx 0.5 \%$ with $\alpha=0.98$ and close to zero for smaller $\alpha$.
} 
between the different FIs/RNDs. In Figure 4 (Appendix) four examples of the dynamically estimated Spearman correlations are shown. It can be clearly seen, that, except for the correlation between JPM and LEH, all time series sharply increase during the year 2007 while they were moving in quite moderate cycles in previous years.

The third step consists of estimating the copula density function $c\left(u_{1}, \ldots, u_{u_{n}}\right)$ on basis of the calculated Spearman correlation coefficients. To guarantee that the $n$ marginals of $c$ are uniform we impose eight moment constraints according to $m_{j}=\frac{1}{1+j}$ onto the marginal of each of the variables $u_{1}, \ldots, u_{n}$. In addition to the constraints on the marginals, $n(n+1) / 2$ Spearman moment conditions are imposed on the estimation of the copula. As the Spearman correlations change every day, we estimate the copula density for each day anew.

In order to estimate the copula density the $n$-dimensional integral in the objective function (12) has to be solved numerically. The optimization with regard to the Lagrange multipliers requires a very precise computation of the integrals which cannot be achieved with ordinary Monte Carlo methods. Therefore, we use a deterministic quadrature rule suggested in Berntsen and Espelid (1991). The method employs a globally adaptive subdivision scheme and uses a cubature rule for the subregion estimation. ${ }^{16}$ As pointed out in Hahn (2005), the method is very reliable in moderate dimensions but quite slow if applied to more than nine dimensions. For this reason, MADs for the entire sample of FIs were only calculated for March 10, 2008 and September 10, 2008 at which we carry out static risk analyses. For the dynamic evaluation, we restrict our analysis to the eight banks in the 'rescued/acquired' and 'bankrupt' group plus JPMorgan as a representative of the 'surviving' group.

In the fourth and final step we obtain the MADs by combining information about the dependence structure and the marginal RNDs using Sklar's theorem. Each copula density is integrated to the copula cdf function $\hat{C}$, such that Sklar's theorem can be applied. Then we integrate the FI's RNDs to the cumulative RND $\hat{F}\left(V_{T}\right)$ and use all of them as arguments in $\hat{C}$. We then obtain the MAD $\hat{H}$ as:

$$
\hat{H}\left(x_{1}, \ldots, x_{n}\right)=\hat{C}\left(\hat{F}_{1}, \ldots, \hat{F}_{n}\right) .
$$

$\hat{H}$ exhibits marginals and a dependence structure that change dynamically every day.

Figures (5)-(7) (Appendix) illustrate graphically the procedure of the multivariate option iPoD framework by showing exemplarily the RNDs, the time series of Spearman correlations and the resulting MAD for the firm's BSC and LEH on March 10, 2008.

\footnotetext{
${ }^{16}$ The method is available in the statistical software $\mathbf{R}$ using the 'cuhre' algorithm from the package R2Cuba.
} 


\section{Results}

In this section we present the results from the empirical application of our framework to the sample of 13 US major FIs. The informational content of our framework is captured by five different risk measures ('systemic distress indicators') that we obtain from the time series of MADs. We analyse the risk measures statically on March 10, 2008 (five days before the collapse of BSC) and on September 10, 2008 (five days before the insolvency of LEH) and dynamically from January 1, 2007 to September 10, 2008.

\subsection{Systemic Distress Indicators}

Based on our estimation framework described in section 2, we suggest five macro-prudential stability measures in order to evaluate the financial system's resilience. The first indicator consists in bivariate conditional PoDs (CPoDs) which measure the default probability for a particular FI $A$ given that another FI $B$ in the system defaults. The CPoDs of all respective institutes under consideration are summarized in a default distress dependence matrix (DDDM). The CPoDs are formalized as follows:

$$
C P o D(A \mid B)=P\left(V_{A} \leq D_{A} \mid V_{B} \leq D_{B}\right)=\frac{P\left(V_{A} \leq D_{A} \cap V_{B} \leq D_{B}\right)}{P\left(V_{B} \leq D_{B}\right)}
$$

On the same rationale we calculate the bivariate conditional quantile risk (CQR), which gives us the probability that a FI's $(A)$ asset value falls below the unconditional $25 \%$ quantile $\left(Q_{25 \% ; A}\right)$ given that another FI $B$ defaults. ${ }^{17}$ Compared to the event of actual default by FI $A$ as measured by CPoDs, this broader distress measure indicates more sensitively an increase in banks' conditional distress and captures also events as downgrades or large losses in market value. Again, the CQRs for a given sample of FIs are summarized in a quantile distress dependence matrix (Q-DDM) and are defined by:

$$
C Q R(A \mid B)=\frac{P\left(V_{A} \leq Q_{25 \% ; A} \mid V_{B} \leq D_{B}\right)-0.25}{0.75}
$$

We subtract 0.25 to adjust the conditional measure for the $25 \%$ unconditional probability of the asset value to fall below its $25 \%$ quantile, which would result in the case of independence. We further normalize the CQR indicator such that its values are for positive dependence structures $\in[0,1]$.

Third, we estimate the probability that at least one other firm in the sample defaults given

\footnotetext{
${ }^{17}$ Note that our estimation approach allows to specify any continuous quantile desired.
} 
a specific firm $A$ defaults (PAO). This risk indicator measures the impact of a specific FI's default onto the resilience of the other institutes in the system. It can be regarded as a measure of the systemic relevance of a particular FI. ${ }^{18}$

$$
P A O=P\left(\text { at least one other FI defaults } \mid V_{A} \leq D_{A}\right) .
$$

The average over the PAOs of all FIs in the sample we refer to as the Financial Interconnectedness Index (FII) and can be regarded as a proxy for the degree of system wide inter-linkage.

Fourth, we evaluate the vulnerability of a FI $A$ to systemic default events (D-VSE). That means, the sensitivity of a certain FI (measured in terms of CPoD) given that at least one FI of the system defaults. This measure is given by the following equation: ${ }^{19}$

$$
D-V S E=P\left(V_{A} \leq D_{A} \mid \text { at least one other FI defaults }\right)
$$

Our fifth risk measure considers the vulnerability of a specific firm $A$ to systemic default events in terms of conditional quantile risk (Q-VSE). The quantile based definition of the VSE provides a broader definition of distress than the CPoD based analogue. Its normalized version is given by:

$$
Q-V S E=\frac{P\left(V_{A} \leq Q_{25 \% ; A} \mid \text { at least one other FI defaults }\right)-0.25}{0.75} .
$$

As a proxy for the system wide level of distress we define the Financial Vulnerability Index (FVI), more precisely the D-FVI and Q-FVI, which are calculated as the averages of the D-VSEs and Q-VSEs over all FIs in the sample, respectively.

While the D-DDM and the PAO are risk measures that were suggested in Segoviano and Goodhart (2009), the remaining distress measures are to our knowledge newly introduced to the literature.

\footnotetext{
${ }^{18}$ Example of PAO calculation in the case of three FIs $A, B, C$ :$$
P A O(A)=P\left(V_{B} \leq D_{B} \cup V_{C} \leq D_{C} \mid V_{A} \leq D_{A}\right) .
$$

${ }^{19}$ Example of D-VSE calculation in the case of three FIs $A, B, C$ :

$$
D \text { - } V S E(A)=P\left(V_{A} \leq D_{A} \mid V_{B} \leq D_{B} \cup V_{C} \leq D_{C}\right) .
$$
}




\subsection{Static Analysis}

Table 1 shows the bivariate CPoDs for all FIs in our sample five days before the bankruptcy of Lehman Brothers (September 15, 2008), summarized by the default distress dependence matrix (D-DDM). Given the default of a FI in the column, the D-DDM provides the probability that a FI in the row defaults between now and time to maturity of the underlying options (theoretically seven month). Looking at the table from a row perspective we learn how sensitive a specific institute is to defaults of the other FIs. From the column perspective we learn about the impact of a specific FI's default on the remaining institutes. Hence, the row averages presented in the last column of the D-DDM can be regarded as a proxy for the (average) vulnerability of a particular FI to distress in one of the other FIs, and the column averages, presented in the last row of the D-DDM, as a proxy for the (average) systemic impact/importance of a specific FI's default on the other institutes.

Starting with the row averages, we see that the group of bankrupt FIs (see section 3), LEH and WM, exhibits by far the highest sensitivities to distress in other institutes $(65.10 \%$ and $81.27 \%$ ), while the institutes which weathered the financial crisis comparably well (surviving FIs: GS, WFC, BAC, JPM, MS) have by far the lowest sensitivities with values ranging from $0.66 \%$ for GS to $4.28 \%$ for MS. The group of acquired/rescued institutes exhibits vulnerabilities that are much lower than for the bankrupt FIs, but much higher than for the surviving FIs (from $8.13 \%$ for C to $28.97 \%$ for WB). From the column averages one can see that the systemic impact of the bankrupt FIs is the lowest among all institutes in the sample, while the acquired/rescued FIs are identified as the institutes with the highest systemic importance with $\mathrm{C}$ as the most important. Taking into account only the 'troubled' FIs (bankrupt/acquired/rescued) we find an inverse relationship between systemic importance and the level of vulnerability of the institutes (see highlights in the table). This might be explained by implicit government guarantees conceded to the systemically important FIs and anticipated by the investors at the option market. Finally, in line with the results above, one finds in the D-DDM, that the troubled FIs were the ones that were the most sensitive to the default of LEH.

In the appendix one can find in Table 8 the quantile distress dependence matrix (Q-DDM) which depicts the bivariate CQRs for the banks in our sample on September 10, 2008; in the Tables 9 and 10 one finds the D-DDM and Q-DDM five days prior to BSC's acquisition by JPM on March 15, 2008. The interpretation of the values of the Q-DDMs are as follows: Given the default of the bank in the column, the Q-DDM provides the probability that the row bank's asset value falls below its unconditional 25\% quantile between now and time to maturity of the underlying option. We will not discuss the results of the Tables 8-10 in 


\begin{tabular}{l|ccccccccccc|c}
\hline & GS & WFC & C & BAC & JPM & AIG & MS & LEH & WB & MER & WM & Row $\varnothing$ \\
\hline GS & 100 & 0.63 & 0.70 & 0.74 & 0.60 & 0.66 & 0.71 & 0.64 & 0.68 & 0.70 & 0.53 & 0.66 \\
WFC & 2.59 & 100 & 2.83 & 2.73 & 2.18 & 2.62 & 2.75 & 2.30 & 2.75 & 2.80 & 2.06 & 2.56 \\
C & 7.73 & 5.47 & 100 & 8.60 & 5.35 & 10.03 & 9.98 & 6.43 & 10.79 & 10.86 & 6.08 & 8.13 \\
BAC & 2.70 & 2.00 & 3.26 & 100 & 1.92 & 3.11 & 3.01 & 2.37 & 3.09 & 3.24 & 1.89 & 2.66 \\
JPM & 1.16 & 1.23 & 1.56 & 1.47 & 100 & 1.51 & 1.58 & 1.36 & 1.52 & 1.58 & 1.15 & 1.41 \\
AIG & 33.66 & 17.11 & 33.65 & 27.60 & 17.55 & 100 & 28.57 & 22.30 & 30.61 & 31.71 & 18.35 & 26.11 \\
MS & 4.29 & 3.07 & 5.77 & 4.59 & 3.14 & 4.91 & 100 & 3.51 & 5.08 & 5.31 & 3.10 & 4.28 \\
LEH & 54.37 & 51.24 & 72.62 & 70.84 & 53.89 & 74.50 & 68.93 & 100 & 73.71 & 78.24 & 52.62 & 65.10 \\
WB & 22.82 & 20.58 & 41.01 & 31.37 & 20.28 & 35.03 & 33.73 & 25.22 & 100 & 37.94 & 21.67 & 28.97 \\
MER & 23.59 & 11.51 & 23.01 & 18.17 & 11.54 & 20.03 & 19.50 & 14.89 & 21.02 & 100 & 11.66 & 17.49 \\
WM & 56.43 & 67.66 & 96.04 & 83.79 & 66.82 & 90.09 & 89.00 & 77.48 & 92.99 & 92.43 & 100 & 81.27 \\
\hline Col. $\varnothing$ & 20.93 & 18.05 & 28.05 & 24.99 & 18.33 & 24.25 & 25.78 & 15.65 & 24.22 & 26.48 & 11.91 & 21.69 \\
\hline
\end{tabular}

Table 1: Default distress dependence matrix (D-DDM) at September 10, 2008. CPoDs of banks in rows given default of banks in columns (in \%).

detail as the upcoming results for the PAO and VSE measures provide qualitatively the same insights that can be obtained from these tables. Compared to the column- and row averages of the DDMs, the PAOs and VSEs are more precise measures of systemic relevance and financial vulnerability of a FI as they take into account all possible systemic events including cascade effects. ${ }^{20}$.

We start by looking at the PAO estimates five days prior to the BSC (Table 2) and the LEH 'event' (Table 3).

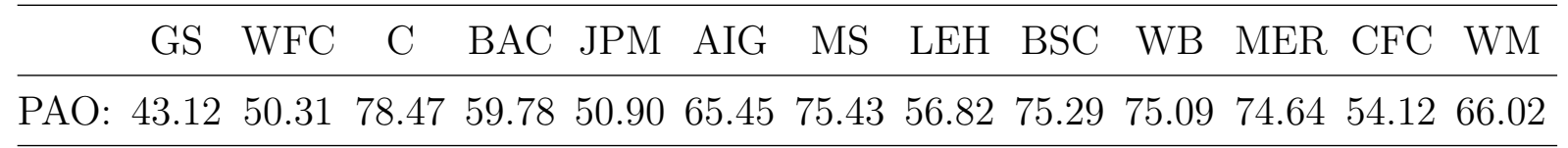

Table 2: Probability that at least one other firm in the sample defaults given a specific firm defaults (PAO) at March 10, 2008 in percentages (average 63.50).

\footnotetext{
${ }^{20}$ The PAO corresponds to the sums of the respective columns of the DDM corrected for 'overlapping' events (e.g. two FIs default at the same time). The VSE corresponds to the sum over the respective rows of the DDM corrected for 'overlapping' events in the conditioning set.
} 


\begin{tabular}{|c|c|c|c|c|c|c|c|c|c|}
\hline GS & WFC & $\mathrm{C}$ & BAC & JPM & AIG & MS & LEH & WB & MER WM \\
\hline 81.12 & 77.19 & 860 & 92.16 & 77.73 & 2678 & 9455 & 8 & $978^{\circ}$ & 12 \\
\hline
\end{tabular}

Table 3: Probability that at least one other firm in the sample defaults given a specific firm defaults (PAO) at September 10, 2008 in percentages (average 87.39).

Most strikingly, the PAO levels of all FIs except for WM rose sharply from March 10, 2008 to September 10, 2008 (in average $\approx 24$ percentage points). This indicates the growing tension and interconnectedness in the US financial sector closer to LEH's bankruptcy. Whereas BSC had an above average systemic relevance five days prior to its acquisition, LEH's systemic importance five days before its collapse is below average. However, given that the PAO of LEH on September 10, 2008 is higher than for any FI on March 10, 2008, gives a first indication for the extreme level of interconnectedness prevailing in the sector prior to the insolvency of LEH. In line with the results of the D-DDM (Table 1), the PAOs identify the rescued/acquired FIs on September 10, 2008 as systemically most important institutes while the bankrupt FIs exhibit PAOs below average. Interestingly, the group of acquired/rescued institutes, including BSC, already show above average PAO values on March 10, 2008.

The Tables 4 to 7 depict the D-VSEs and Q-VSEs, for March 10, 2008 and September 10, 2008 respectively.

\begin{tabular}{cccccccccccccc}
\hline GS & WFC & C & BAC & JPM & AIG & MS & LEH & BSC & WB & MER & CFC & WM \\
\hline D-VSE: & 0.40 & 2.61 & 10.04 & 1.24 & 2.82 & 2.95 & 3.50 & 5.77 & 7.27 & 5.06 & 4.86 & 53.55 & 37.05 \\
\hline
\end{tabular}

Table 4: Vulnerability of a firm to systemic default events (D-VSE) at March 10, 2008 in percentages (average 10.55).

\begin{tabular}{lccccccccccc}
\hline GS & WFC & C & BAC & JPM & AIG & MS & LEH & WB & MER & WM \\
\hline D-VSE: & 0.51 & 1.97 & 5.27 & 1.74 & 1.09 & 17.15 & 2.63 & 52.47 & 19.81 & 10.81 & 78.99 \\
\hline
\end{tabular}

Table 5: Vulnerability of a firm to systemic default events (D-VSE) at September 10, 2008 in percentages (17.50).

We first take a look at the D-VSE tables. Equivalently to the results in Table 1, on September 10, 2008 the bankrupt FIs have by far the highest values, the 'safe' institutes by far the lowest, and the rescued/acquired FIs values of medium size. Comparing D-VSEs and PAOs 
for the 'troubled' FIs, we find again an inverse relationship between systemic importance and (assessed) financial soundness/vulnerability. The D-VSEs increase in average from March 10, 2008 to September 10, 2008, which shows the decreasing financial soundness in the financial sector in the course of 2008. Already in March most of the 'troubled' institutes, including BSC and especially CFC, exhibit larger values than the 'safe' FIs. However, except for CFC, WM and $\mathrm{C}$ the classification is less clear than in September. The relatively small D-VSE value for BSC five days prior to its acquisition might be explained by the relatively high systemic importance implied by its PAO.

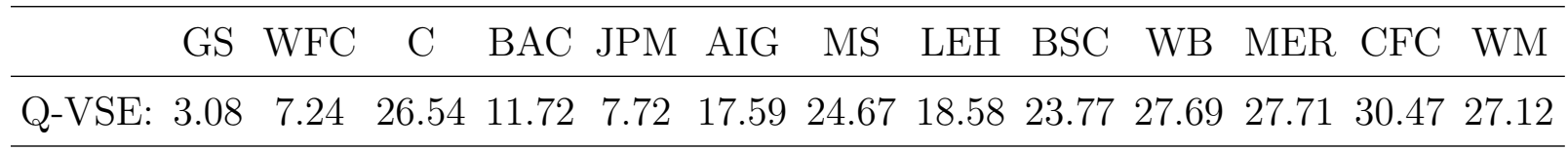

Table 6: Vulnerability of a specific firm to systemic default events in terms of conditional quantile risk (Q-VSE) at March 10, 2008 in percentages (average 19.53).

\begin{tabular}{|c|c|c|c|c|c|c|c|c|c|c|}
\hline GS & WFC & $\mathrm{C}$ & $\mathrm{BAC}$ & JPM & AIG & MS & LEH & WB & MER & WM \\
\hline -VSE: 12.54 & 6.12 & J. & 20.53 & 17.2 & 34.0 & 21.9 & 30.0 & 276 & 36.01 & \\
\hline
\end{tabular}

Table 7: Vulnerability of a specific firm to systemic default events in terms of conditional quantile risk (Q-VSE) at September 10, 2008 in percentages (average 27.28).

The picture becomes clearer if we look at the Q-VSEs in the Tables 6 and 7. From a theoretical point of view we expect that the Q-VSEs do not discriminate between systemically relevant and less relevant FIs, as the likelihood for large losses in market value and downgrades should not be affected by implicit governmental bailout guarantees. The obtained results confirm these expectations as the Q-VSE for the 'troubled' FIs are very similar to each other and hence compared to the D-VSEs, where the values between the bankrupt and acquired/rescued FIs differed largely, converge. ${ }^{21}$ Consequently, FIs with comparably low D-VSEs but high Q-VSEs are a clear indication for implicit bailout guarantees for these institutes. Following this reasoning, we find on September 10, 2008 bailout guarantees for C, AIG, WB and MER as they exhibit Q-VSEs similar to the ones of the 'bankrupt' FIs, WM and LEH, but much lower D-VSEs. On March 10, 2008 we find bailout guarantees for C, MS, WB, MER and $\mathrm{BSC}^{22}$ since their D-VSEs are much lower than those of the high risk firms CFC and WM

\footnotetext{
${ }^{21}$ This convergence can also be found for the row averages of the Q-DDM in Table 8 (Appendix).

${ }^{22}$ From Table 10 (Appendix) one can learn that all of the 'troubled' FIs were highly sensitive to the default of BSC, which indicates that these FIs had similar exposures as BSC and/or were strongly linked to this firm.
} 
(according to D-VSE) but their Q-VSEs are similar.

Besides helping to identify bailout guarantees, the Q-VSEs further provide sharper and more timely discrimination between 'safe' and troubled FIs as the D-VSEs do. On September 10, 2008 all 'troubled' FIs exhibit above average Q-VSE values, while all 'safe' FIs have below average values. On March 10, 2008 all 'troubled' institutes, including BSC, show above- or close-to-average values, while most 'safe' FIs (except MS) show clear below-average Q-VSEs. In summary, the main findings of the static systemic risk analysis are:

- The D-VSEs clearly discriminate between the three groups: 'bankrupt', 'acquired/rescued' and 'surviving' FIs .

- The 'acquired/rescued' FIs exhibit lower D-VSEs than the 'bankrupt' FIs but higher D-VSEs than the 'surviving' FIs.

- The 'acquired/rescued' FIs are found to be more systemically important (as measured by the PAOs) than the 'bankrupt' FIs.

- A comparison of D-VSEs and Q-VSEs gives clear indications for implicit bailout guarantees conceded to the 'acquired/rescued' FIs, since the indicated risk levels for the 'bankrupt' and for the 'rescued/acquired' firms converge in the case of Q-VSEs.

- The Q-VSEs are able to identify the 'troubled FIs' more clearly at an early stage.

\subsection{Dynamic Systemic Risk Analysis}

For the dynamic analysis of our risk indicators we estimate time series for the PAOs, DVSEs and Q-VSEs from January 1, 2007 to September 10, 2008. Du to the problems with high-dimensional integration (see section 4) we restrict our sample to the 'troubled' FIs plus JPM as representative of the 'surviving' FIs.

Figure 1.(a) shows exemplarily the time series of PAOs (in percentages) for C, LEH and WM. All PAOs start to increase sharply on July 30, 2007 and rise relatively continuously until September 10, 2008. There are two periods of comparative recovery, the first one from January 11, 2008, when BAC announced the acquisition of CFC, to the end of February and the second one from March 15, 2008, when JPM announced the acquisition of BSC, until the end of April. Interestingly, WM has relatively high systemic importance until January 2008. This is due to its high connectedness with CFC (see also Table 9 (Appendix)) but after the announced acquisition of CFC by BAC on January 11, 2008, WM's impact decreases and in June 2008 with the looming completion of the takeover of CFC on July 1, 2008 even becomes the least relevant bank in the sample. In contrast, $\mathrm{C}$ is regarded as the most important FI 
throughout the whole year of 2008, with LEH and WM not even coming close.

However, as already suspected in the static analysis, the historically extreme PAO levels, observable since at least November 2007, even for the less important FIs (WM and LEH) give clear indications that the collapse of any bank in our sample will have an immense impact on the stability of the financial sector. This impression is strongly backed by Figure 1.(b) which shows the evolution of the Financial Interconnectedness Index (FII) over time. The FII is calculated as the average over the PAOs of all FIs in the sample (see Figure 8 (Appendix) for the PAO time series of all considered FIs) and can be seen as a proxy for the degree of interconnectedness prevailing in the financial sector. We see that the degree of interconnectedness in the sector reaches historically unseen levels starting in November 2007, and especially since June 2008. This strongly suggests a 'too interconnected too fail' problematic prevailing in the sector also around the time of LEH's collapse in September 2008, which implies that the FED's decision to let LEH go bankrupt might have been correct from a cross-dimensional but not from a time-dimensional perspective.

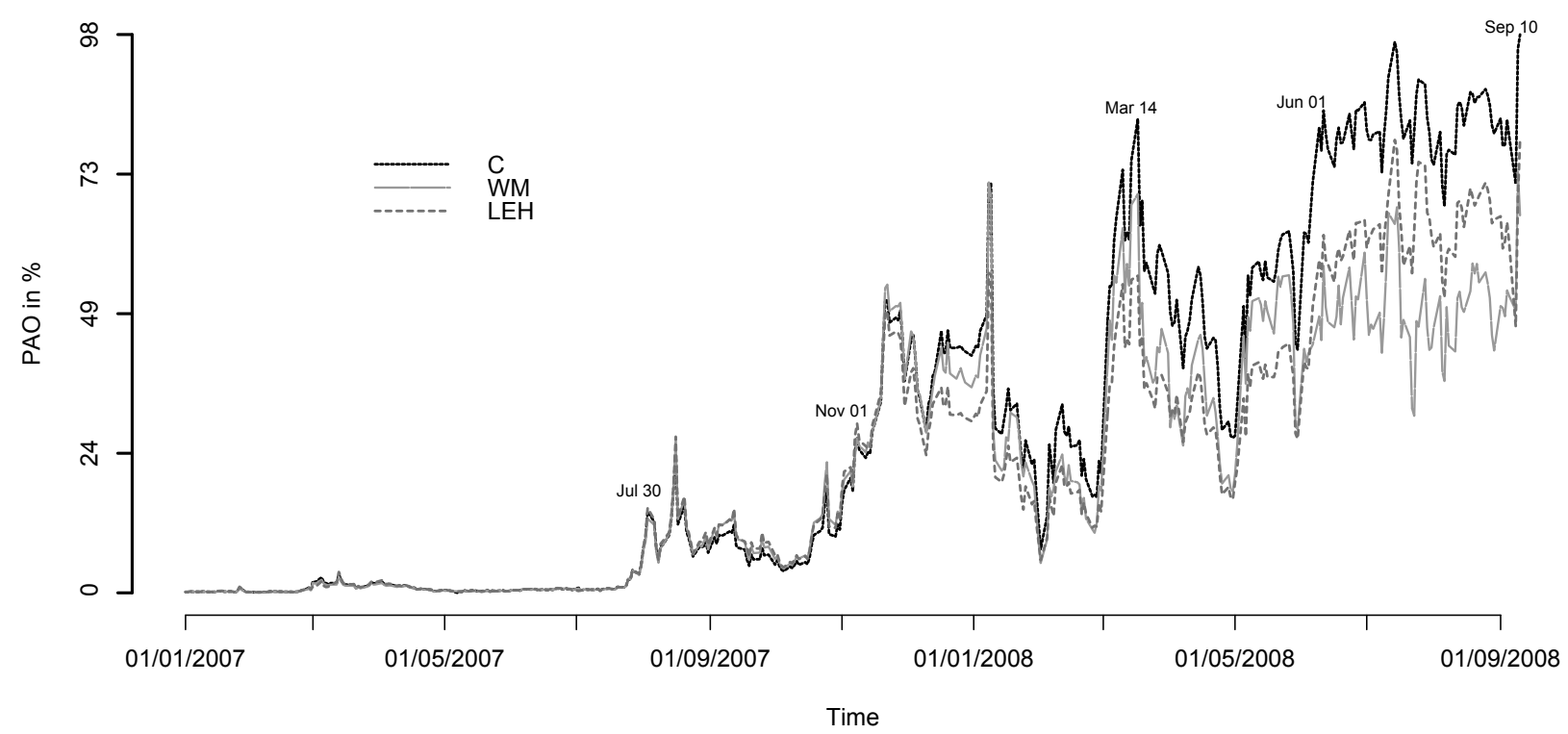

Figure 1.(a): Probability that at least one other firm in the sample defaults given a specific firm defaults (PAO) of C, LEH and WM from January 2007 to September 10, 2008 (in \%). 


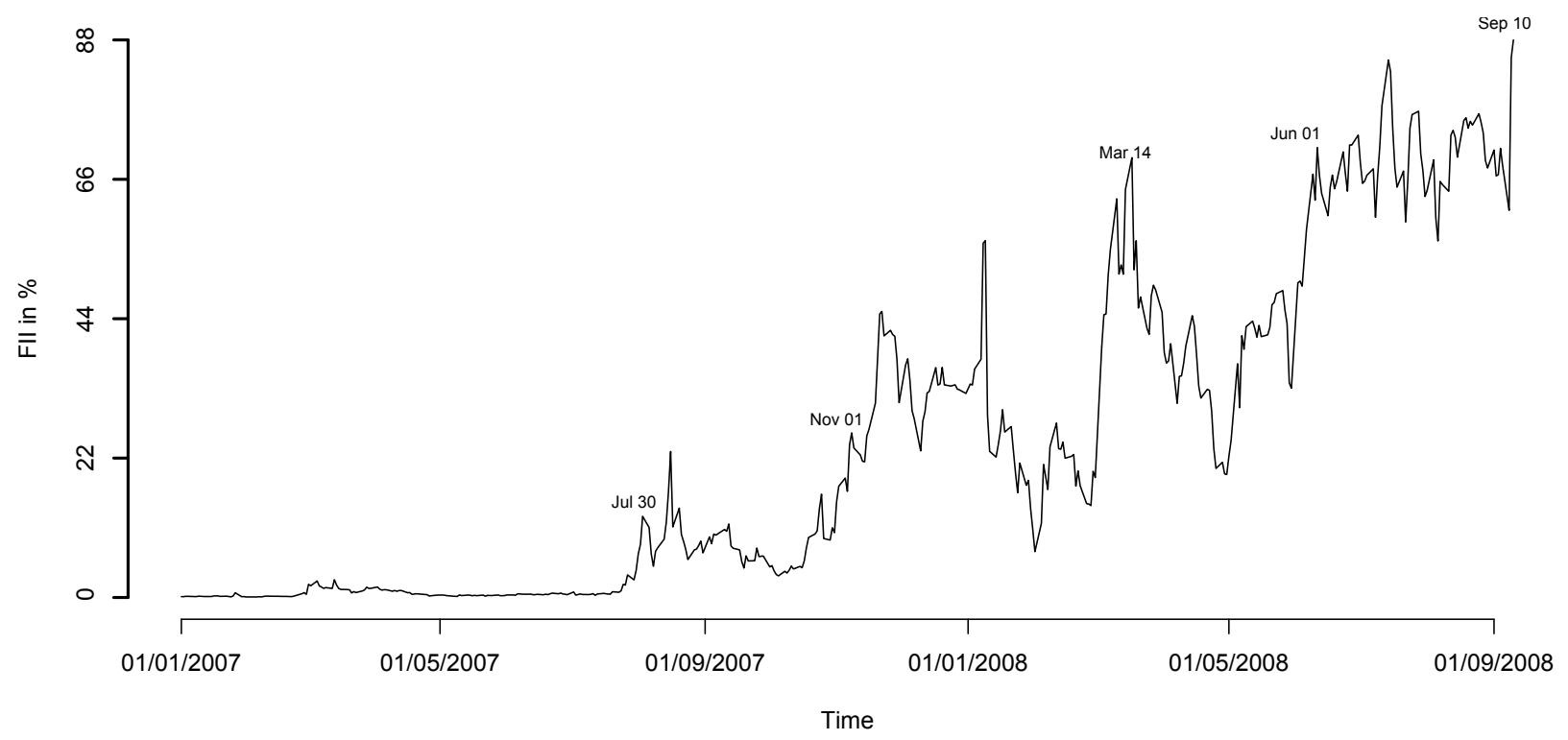

Figure 1.(b): Financial Interconnectedness Index (FII) from January 2007 to September 10, 2008 (in \%).

Figure 2.(a) shows the time series of D-VSEs for C, BSC, LEH and JPM from January 2007 to September 10, 2008. The D-VSEs start to increase at the end of July 2007, and in November 2007 we see the beginning divergence between the time series of the 'troubled' FIs, C, BSC and LEH, and the time series of JPM (as representative of the 'safe' FIs). This divergence reaches extreme levels in the weeks previous to BSC's collapse in mid-March 2008 and remains high also in the post-BSC period. In May 2008 also the time series of LEH and $\mathrm{C}$ also begin to diverge strongly which gives clear indications that a default is much more likely for LEH than for $\mathrm{C}$ which might be explained by a possible bailout guarantee conceded to $\mathrm{C}$.

In Figure 2.(b) we see the timely evolution of the average value of the D-VSEs for all FIs in our sample (see Figure 9 (Appendix) for the VSE time series of all considered FIs), which we refer to as the Default Financial Vulnerability Index (D-FVI). The increasing financial distress in the sector is displayed as early as July 30, 2008 and rises similarly to the PAOs above continuously until September 10, 2008.

Figure 3.(a) depicts the Q-VSE time series of C, WM, JPM, WB and BSC. Here we see a more clear divergence between the 'safe' bank JPM and the 'troubled' FIs than for the DVSEs. The divergence begins in October 2007 and gets more and more extreme over the rest of the time period. As already suspected in the static analysis we see that the values of the 
'troubled' FIs move very closely to each other over time. Hence, already in the last months of 2007 the group of FIs which faced the most severe problems during the sub-prime crisis can be identified clearly on basis of the Q-VSE measure. This is also true for BSC, whose values rise sharply from November 2007 until its collapse in March 2008. When comparing the time series of Q-VSEs and D-VSEs for the individual banks we find strong indications that the investors at the option markets anticipated the implicit bailout guarantees for $\mathrm{C}$ and BSC since their D-VSE levels did not rise to such extreme levels as seen for LEH, CFC and $\mathrm{WM}^{23}$ (see Figure 9 (Appendix) for the D-VSE time series of the latter two FIs) but their distress levels as measured by the Q-VSEs were very similar (see Figure 10 in the Appendix for Q-VSE time series of CFC and WM). Using the time series in the Appendix (Figure 9 and Figure 10) we further find bailout guarantees for AIG, WB and MER.

Finally, Figure 3.(b) shows the Quantile-Financial Vulnerability Index (Q-FVI) calculated as the average of the Q-VSE of all considered FIs. At an early stage, the Q-FVI shows more distinctly the high degree of distress in the financial sector than the D-FVI, because it rises in a more continuous manner and in a concave rather than in a convex way.

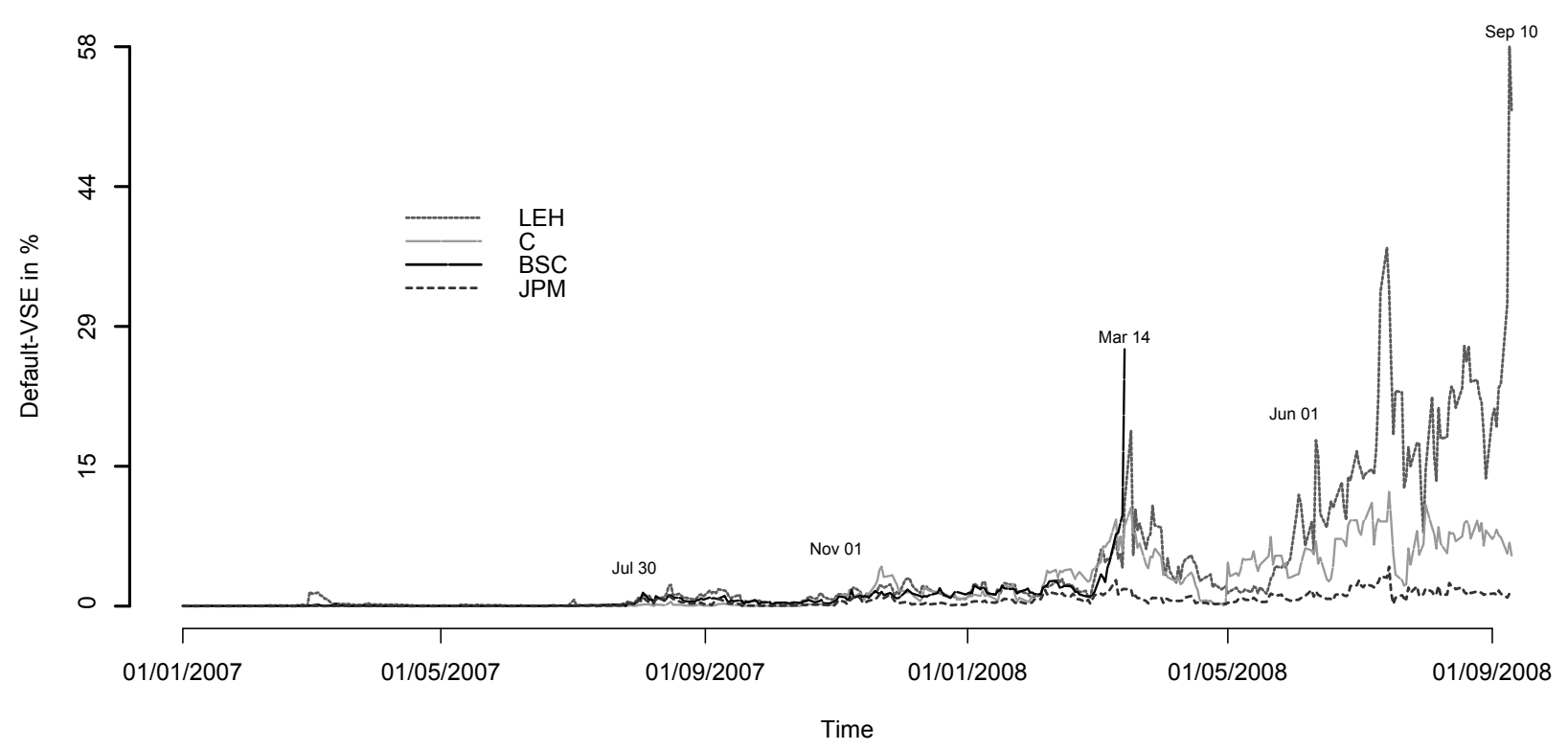

Figure 2.(a): Vulnerability of a firm to systemic default events (D-VSE) of C, JPM, LEH and BSC from January 2007 to September 10, 2008 (in \%).

\footnotetext{
${ }^{23}$ While CFC finally was acquired by BAC on July 1, 2008 and did not go bankrupt like LEH and WM, its high D-VSE levels indicate that CFC did not have an implicit bailout guarantee.
} 


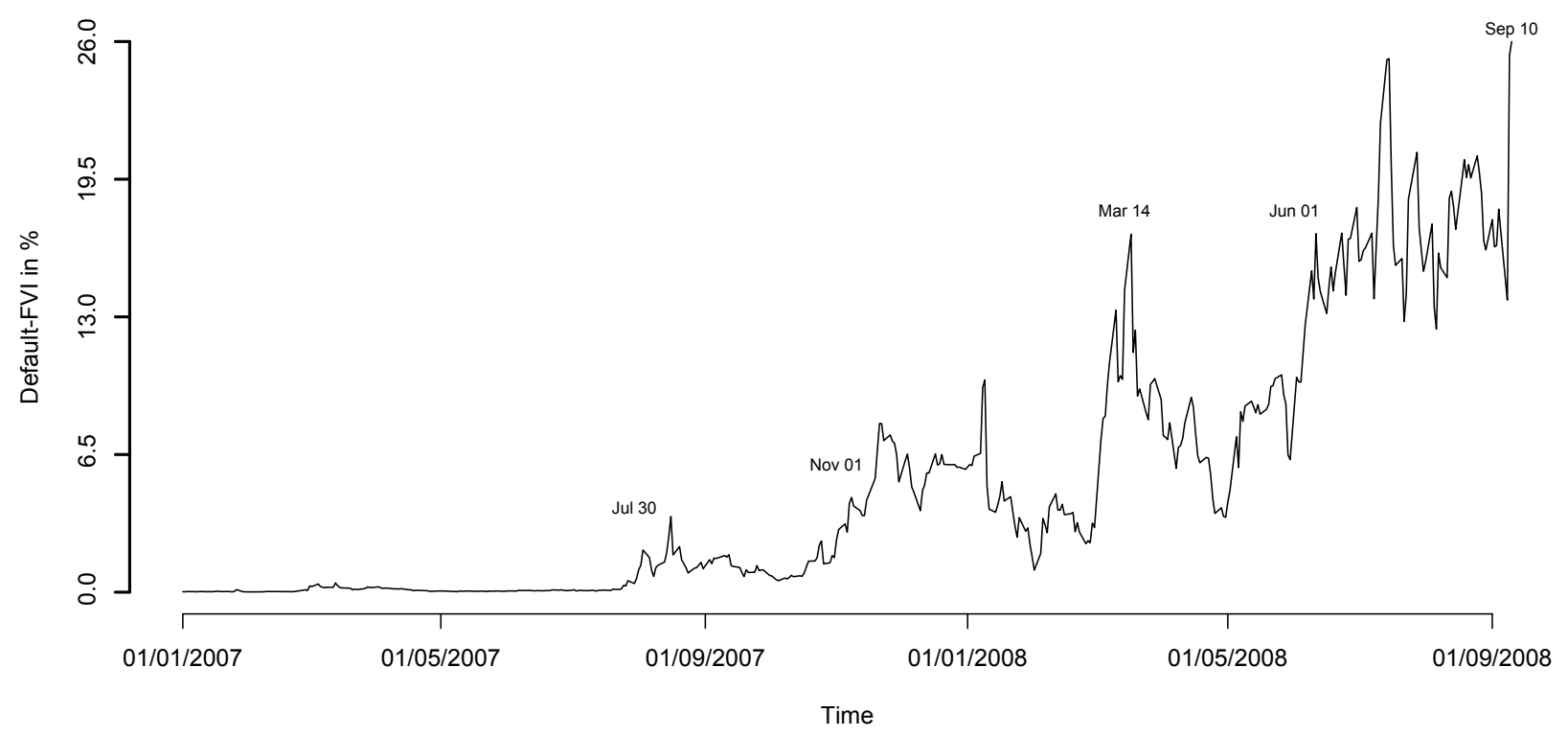

Figure 2.(b): Default Financial Vulnerability Index (D-FVI) of C, AIG, LEH and JPM from January 2007 to September 10, 2008 (in \%).

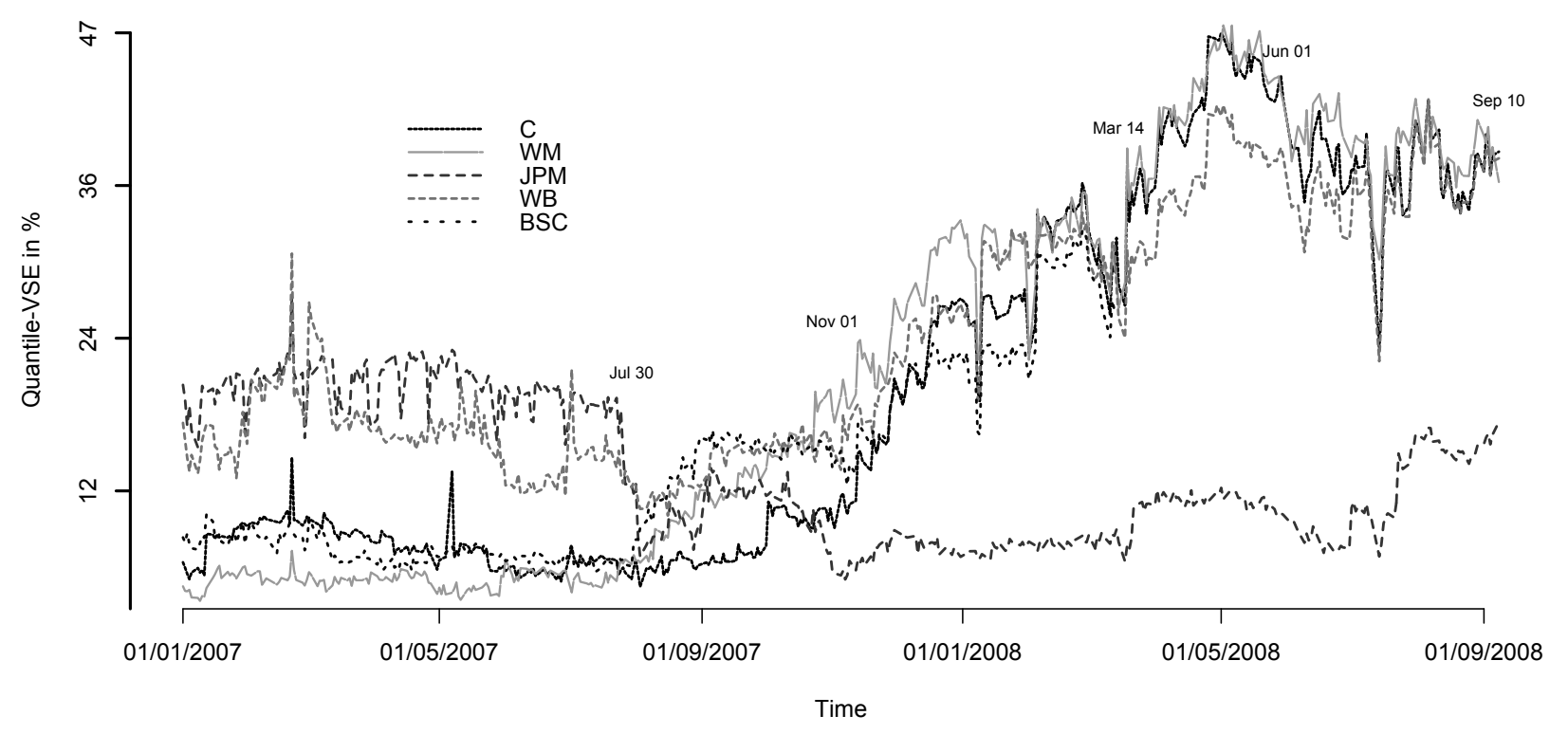

Figure 3.(a): Vulnerability of a specific firm to systemic default events in terms of conditional quantile risk (Q-VSE) of C, AIG, LEH and JPM from January 2007 to September 10, 2008 (in \%). 


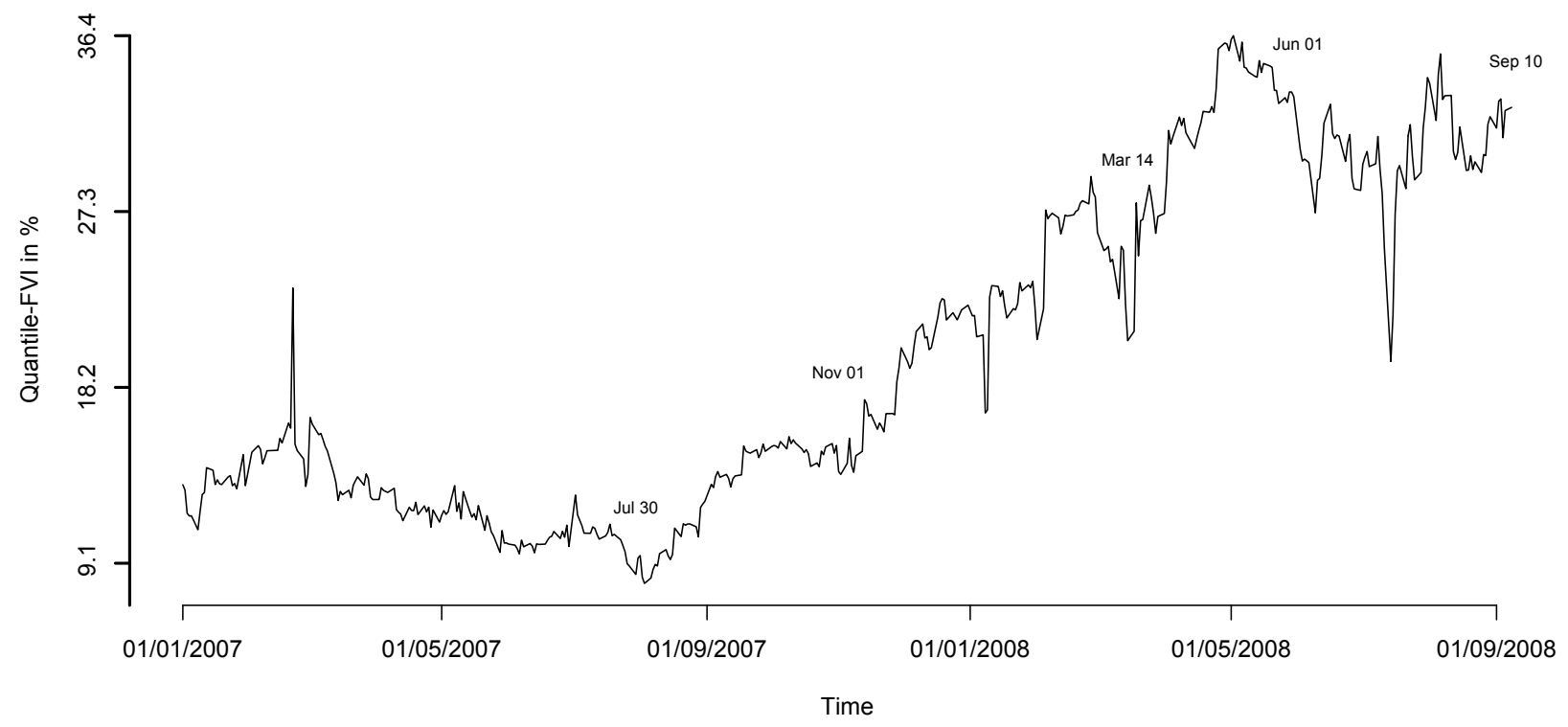

Figure 3.(b): Quantile Financial Vulnerability Index (Q-FVI) of C, AIG, LEH and JPM from January 2007 to September 10, 2008 (in \%).

The main insights from the dynamic risk analysis can be summarized as follows:

- The PAO levels indicate a 'too interconnected too fail' dilemma since November 2007, as even the least systemically important FIs exhibited within an historical context extreme values.

- All indicators (PAOs, D-VSEs, Q-VSEs) begin to sharply increase in July 2007.

- The discrimination/identification between 'troubled' and 'safe' FIs happens as early as November 2007 (for CFC even in July 2007) and is highly stable over time.

- At an early stage of distress the Q-VSEs identify more clearly the 'troubled' FIs than the D-VSEs.

- The Q-VSEs of all 'troubled' FIs exhibit in a stable manner similar distress levels and hence, by comparing the displayed riskiness of D-VSE and Q-VSE for the individual FIs, we obtain timely indications for implicit bailout guarantees.

- The derived indices FII, D-FVI and Q-FVI are able to display the increasing degree of distress and interconnectedness in the financial sector over the period of July 2007 to September 2008. The Q-FVI hereby indicates more distinctly at an early stage the extreme degree of distress as it increases more continuously than the other indices and 
in a concave rather than in a convex way.

\section{Conlusion}

In this paper we suggested a novel framework to assess systemic risk in financial sectors. Our framework uses daily option prices to estimate time series of multivariate (risk neutral) asset distributions (MADs) for a sample of financial institutions (FIs). The MADs are obtained by combining the (univariate) option iPoD approach of Capuano (2008) and Vilsmeier (2011) with the most entropic copula methodology of Chu (2011). While the option iPoD procedure provides us with time series of the individual FI's asset distributions (RNDs) and their probabilities of default (PoDs), the MEC methodology allows us to estimate a copula for the multivariate asset distribution on basis of Spearman rank correlations that we calculate from the RND time series. Using Sklar's theorem we combine the RND time series and the copula to obtain the MADs. For the estimation of the RNDs neither balance sheet data nor recovery rate assumptions are required as the option iPoD framework derives the individual bank's asset distributions in a purely statistical way solely using daily sets of equity option prices. The Spearman rank correlations are estimated dynamically with exponentially decreasing influence of past observations such that the dependence structure of the MAD described by the copula may change every day. The dynamic rank correlations measure linear as well as non-linear dependence structures and can perfectly capture the increasing correlations during times of economic downturns that are usually detected.

Both, the RNDs and the copula are estimated using a semi-parametric estimation procedure based on the entropy function. The framework provides smooth density estimates that are highly flexible with regard to their functional forms and MADs whose marginals (RNDs) and dependence structure is updated on a daily basis.

Time series of MADs were estimated for 13 major US-FIs during the period of the US subprime crisis from January 2007 to September 2008. On basis of the MADs we derived five different systemic risk indicators which are based on conditional PoDs and conditional lower quantiles. The indicators were analyzed statically on March 10, 2008 and September 10, 2008 as well as dynamically over the whole sample period and provide strong evidence for the high informational content resulting from our estimation approach. The static analysis showed that the derived indicators are able: i) to clearly discriminate between the institutes that had the most severe problems during the financial crisis ('troubled' FIs) and those who weathered the turmoil comparably well ('safe' FIs), ii) to distinctly classify the 'troubled' FIs into the group of institutes that were acquired or rescued during the crisis and those 
banks that went bankrupt, iii) to show that the acquired/rescued FIs were assessed as more systemically important than the bankrupt FIs and iv) to provide strong evidence for implicit bailout guarantees conceded to the systemically most important institutes. From the dynamic analysis we obtain the following insights: i) all indicators begin to sharply increase in July 2007, ii) the discrimination between 'troubled' and safe institutes happens as early as November 2007 (in case of Countrywide Financial (CFC) even in July 2007) and is highly stable over time, iii) the implicit bailout guarantees conceded to some FIs can be identified at an very early stage (differing among institutes) and iv) the degree of dependence among the FIs indicates a severe 'too interconnected too fail' dilemma in the US financial sector since (at least) November 2007.

Future research should intend to minimize the dependence of the estimated correlation coefficients on outdated historical data. Big improvement might be obtained by using intra-day data and realized correlations theory as for instance in Huang et al. (2009). Further, more efficient ways to solve the high-dimensional integrals in the MEC estimation should be found. A valuable step in this direction might be to use the connection between the MEC and the FRAME model (a type of Markov Random Field model) of Zhu et al. (1998) as suggested in Huang and Freedman (2010). 


\section{References}

Alhassid, Y., N. Agmon, and R. D. Levine (1978). An Upper Bound for the Entropy and its Applications to the Maximal Entropy Problem. Chemical Physics Letters 53, 22-26.

Avesani, R., A. Pascual, and J. Li (2006). A New Risk Indicator and Stress Testing Tool: A Multifactor Nth-to-Default CDS Basket. IMF Working Paper 105.

Barndorff-Nielsen, O. and N. Shephard (2004). Econometric Analysis of Realized Covariation: High-Frequency Covariance, Regression and Correlation in Financial Economics. Econometrica 72, 885-925.

Bernanke, B. (2010). Discussion on the Squam Lake Report: Fixing the Financial System. Speech at the Squam Lake Conference, New York.

Berntsen, J. and T. Espelid (1991). An Adaptive Algorithm for the Approximate Calculation of Multiple Integrals. ACM Transactions on Mathematical Software 17(4), 437-451.

BIS (2011). Macroprudential Policy Tools and Frameworks: Progress Report to G20. Technical report, Published jointly with IMF and FSB.

Blest, D. C. (2000). Rank Correlation: An Alternative Measure. Australian and New Zealand Journal of Statistics 42(1), 100-111.

BoE (2009). The Role of Macroprudential Policy. Bank of England Discussion Paper.

Capuano, C. (2008). The Option-iPoD. The Probability of Default Implied by Option Prices based on Entropy. IMF Working Paper 08(194).

Chan-Lau, J. and T. Gravelle (2005). The END: A New Indicator of Financial and Nonfinancial Corporate Sector Vulnerability. IMF Working Paper 231.

Chu, B. (2011). Recovering Copulas from Limited Information and an Application to Asset Allocation. Journal of Banking and Finance 35, 1824-1842.

Cover, T. M. and J. A. Thomas (2006). Elements of Information Theory (2nd ed.). John Wiley \& Sons.

Cox, J. and S. Ross (1976). The Valuation of Options for Alternative Stochastic Processes. Journal of Financial Economics 3, 145-166.

Crosbie, P. and A. Kocagil (2003). Modeling Default Risk. Moody's KMV Technical Paper. 
Frey, R., A. McNeil, and M. Nyfeler (2001). Copulas and Credit Models. Risk 14, 111-114.

Group of Thirty (2010). Enhancing Financial Stability and Resilience: Macroprudential Policy, Tools, and Systems for the Future. The Group of Thirty (G30) Technical Document.

Hahn, T. (2005). Cuba:A Library for Multidimensional Numerical Integration. Computer Physics Communications 168, 78-95.

Huang, J. and C. Freedman (2010). Most Entropic Copulas: General Form, and Calibration to High-Dimensional Data in an Important Special Case. Available at SSRN: http://ssrn.com/abstract $=1725517$.

Huang, X., H. Zhou, and H. Zhu (2009). A Framework for Assessing the Systemic Risk of Major Financial Institutions. BIS Working Papers.

Huang, X., H. Zhou, and H. Zhu (2012). Systemic Risk Contributions. Journal of Financial Services Research 42, 55-83.

Jaynes, E. T. (1957). Information Theory and Statistical Mechanics. Physical Review 106, 620-630.

Jenkins, P. and G. Thiessen (2012). Reducing the Potential for Future Financial Crises: A Framework for Macro-Prundential Policy in Canada. C.D. Howe Institute Commentary 351.

J.P.Morgan (1997). CreditMetrics. Technical Document.

Kullback, S. and R. Leibler (1951). On Information and Sufficiency. Annals of Mathematical Statistics 22, 79-86.

Lehar, A. (2005). Measuring Systemic Risk: A Risk Management Approach. Journal of Banking and Finance 29, 2577-2603.

Matros, P. and J. Vilsmeier (2012). Measuring Option Implied Degree of Distress in the US Financial Sector Using the Entropy Principle. Deutsche Bundesbank Discussion Paper 30.

Merton, R. C. (1974). On the Pricing of Corporate Debt: The Risk Structure of Interest Rates. Journal of Finance 29, 449-470.

Murphy, E. (2013). Financial Stability Oversight Council: A Framework to Mitigate Systemic Risk. CRS Report for Congress. 
Nelsen, R. (2006). An Introduction to Copulas. Springer Science+Business Media, Inc.

RiskMetricsTM (1996). Technical Document, Fourth Edition. Morgan Guaranty Trust Company and Reuters Ltd.

Schmid, F. and R. Schmidt (2007). Multivariate Conditional Versions of Spearman's Rho and Related Measures of Tail Dependence. The Journal of Multivariate Analysis 98, $1123-1140$.

Segoviano, M. A. and C. Goodhart (2009). Banking Stability Measures. IMF Working Paper.

Shannon, C. E. (1948). A Mathematical Theory of Communication. Bell System Technical Journal 27, 379-423.

Sklar, A. (1959). Fonctions de Repartition a n Dimensions et leurs Marges. Publications de l'Institut de Statistique de l'Universite de Paris 8, 229-231.

Tudela, M. and G. Young (2005). A Merton-Model Approach to Assessing the Default Risk of UK Public Companies. Journal of Theoretical and Applied Finance 8, 737-761.

Vilsmeier, J. (2011). Updating the Option Implied Probability of Default Methodology. BGPE Working Paper.

Wheelock, D. C. (2010). Lessons Learned? Comparing the Federal Reserve's Responses to the Crisis of 1929-1933 and 2007-2009. Federal Reserve Bank of St. Louis Review March/April, 89-108.

Zhu, S. C., Y. Wu, and D. Mumford (1998). Filters, Random Fields and Maximum Entropy (FRAME): Towards a Unified Theory for Texture Modeling. International Journal of Computer Vision 27(2), 107-126. 


\section{Appendix}
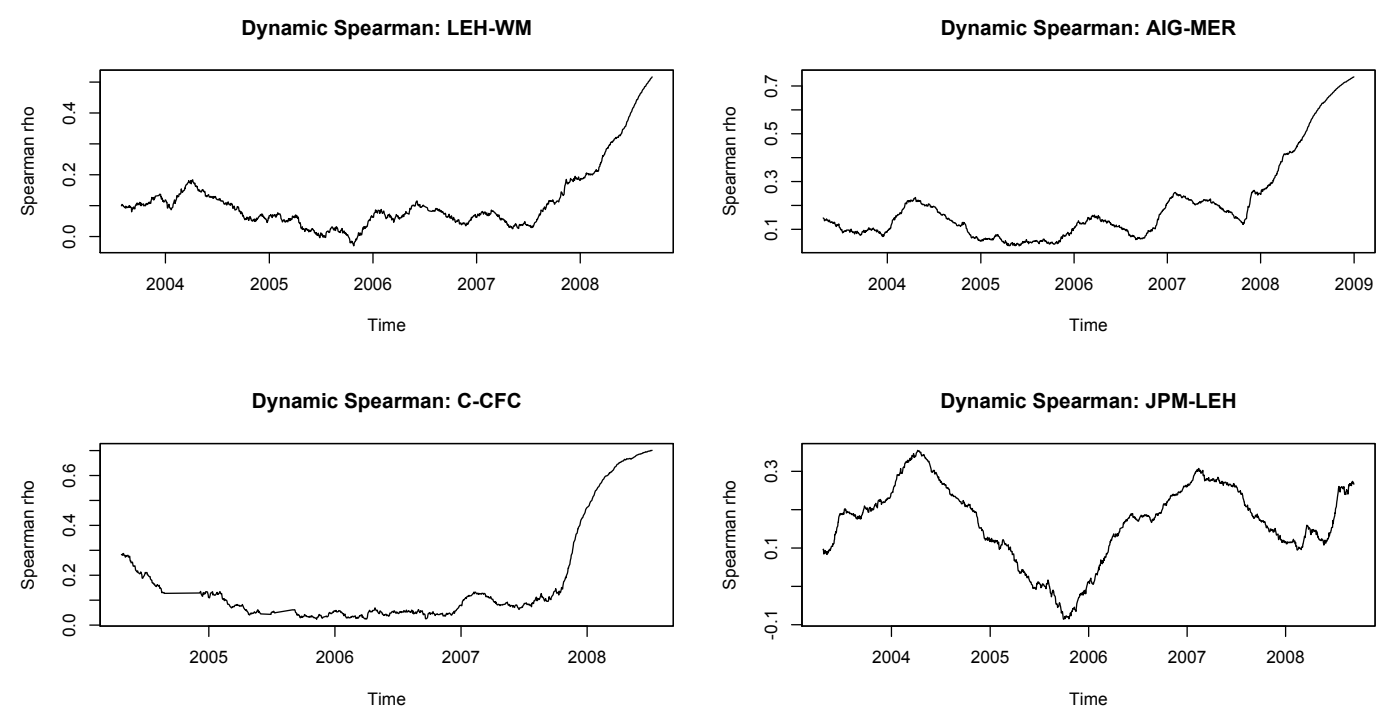

Figure 4: Examples for dynamic Spearman correlations.

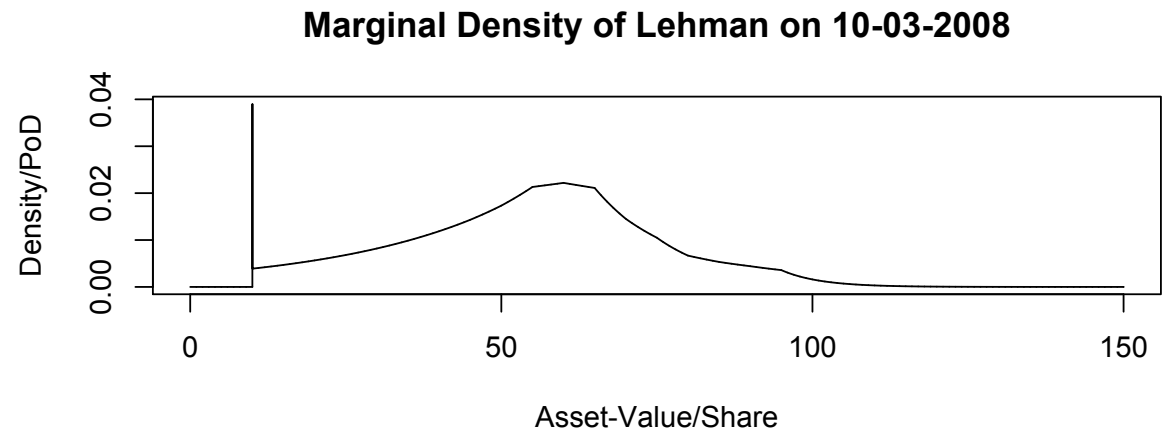

Marginal Density of Bear Stearns on 10-03-2008

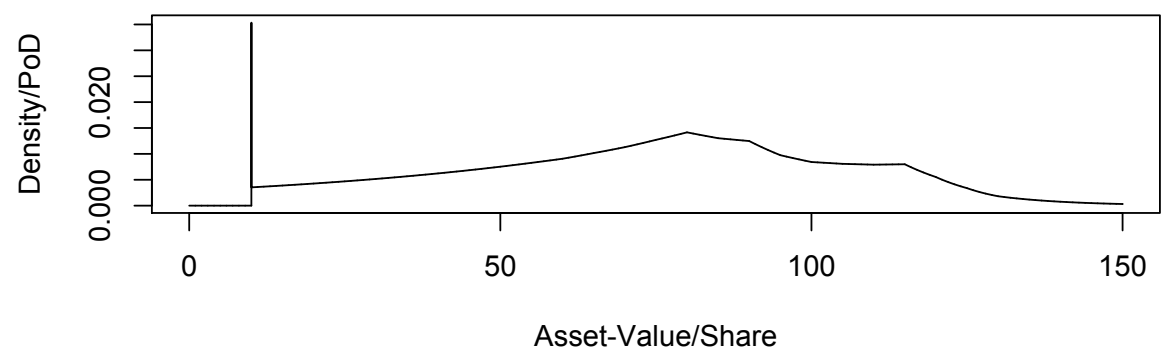

Figure 5: Marginal density functions of Lehman Brothers and Bear Stearns at March 10, 2008. 


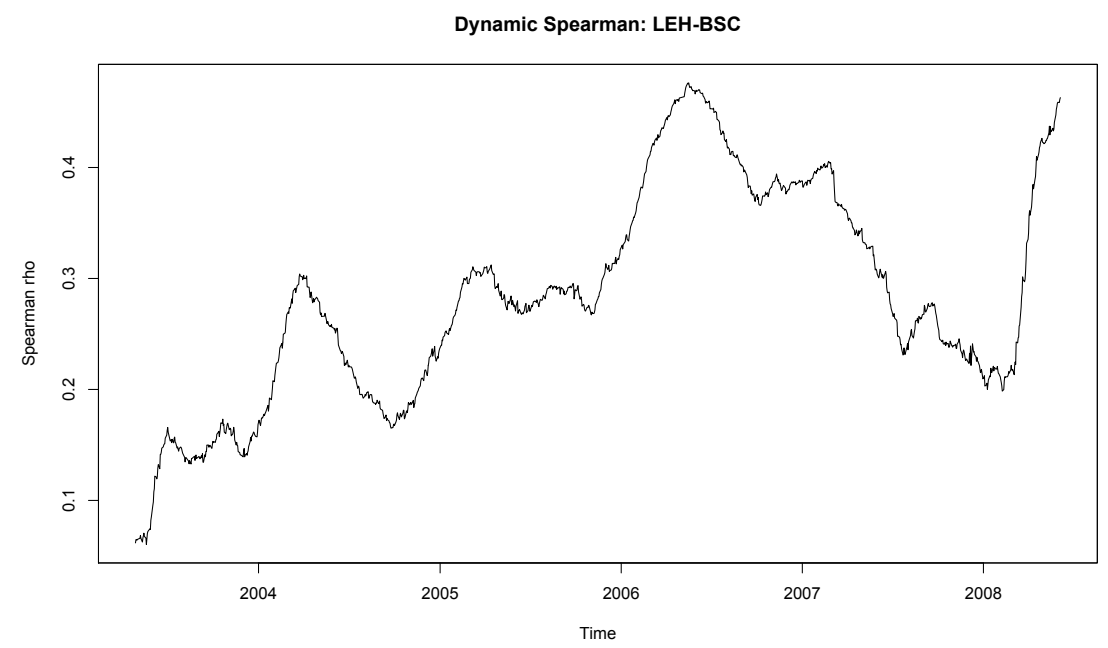

Figure 6: Dynamic Spearman rank correlations $(\alpha=0.99)$ between Lehman Brothers and Bear Stearns from January 1, 2003 to September 10, 2008.

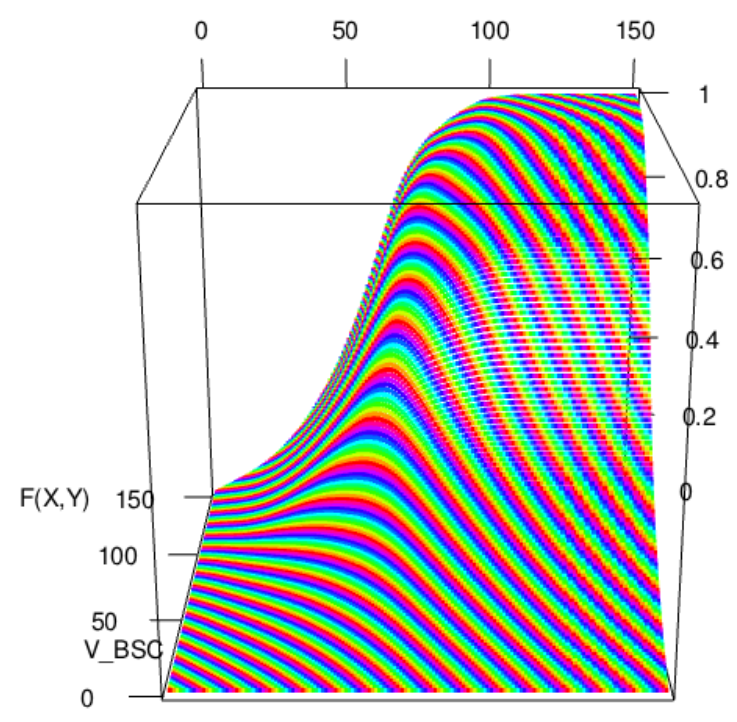

V_LEH

Figure 7: Bivariate cumulative density function of Lehman Brothers and Bear Stearns at March 10, 2008. 


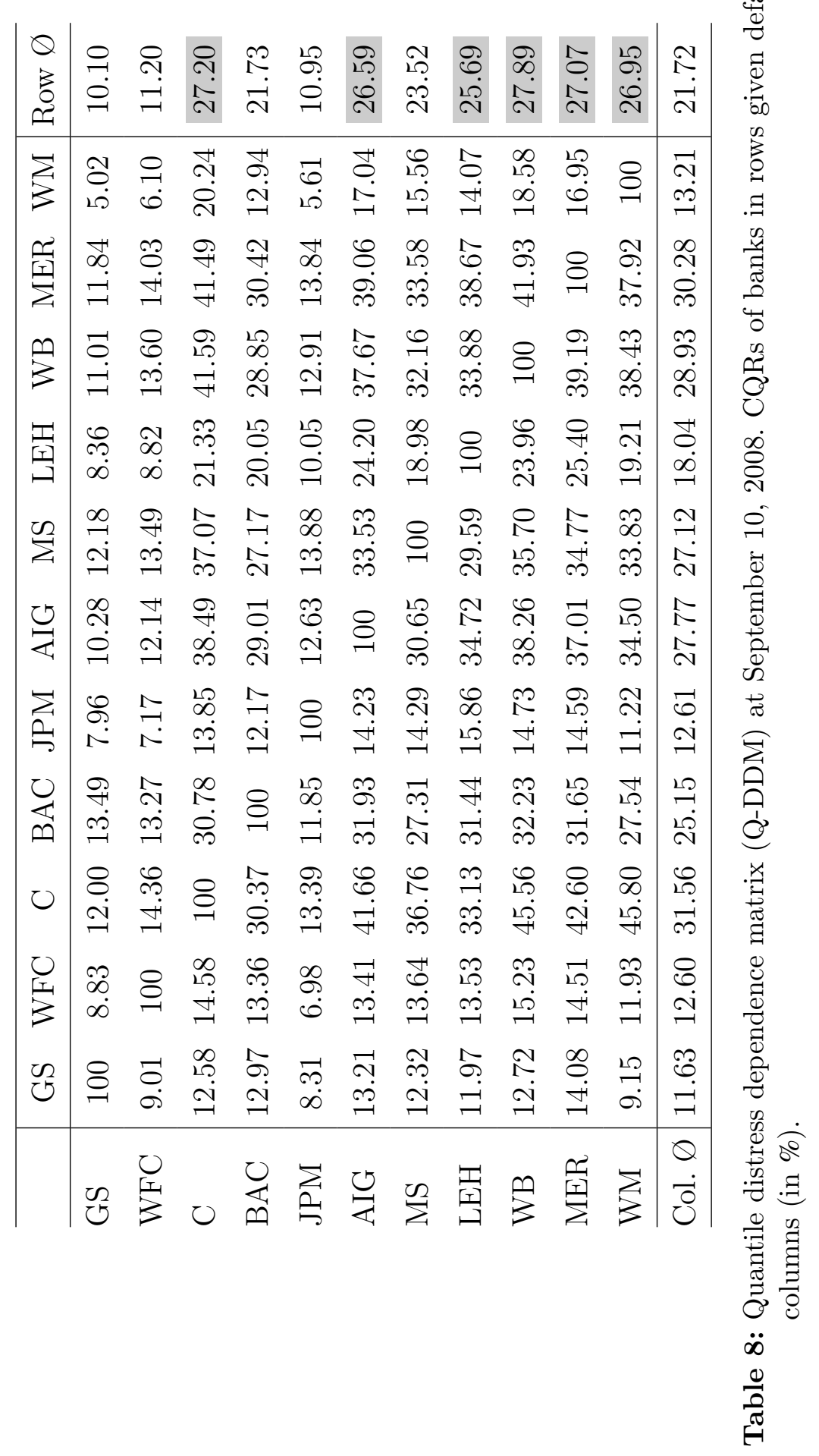

.

䨔 


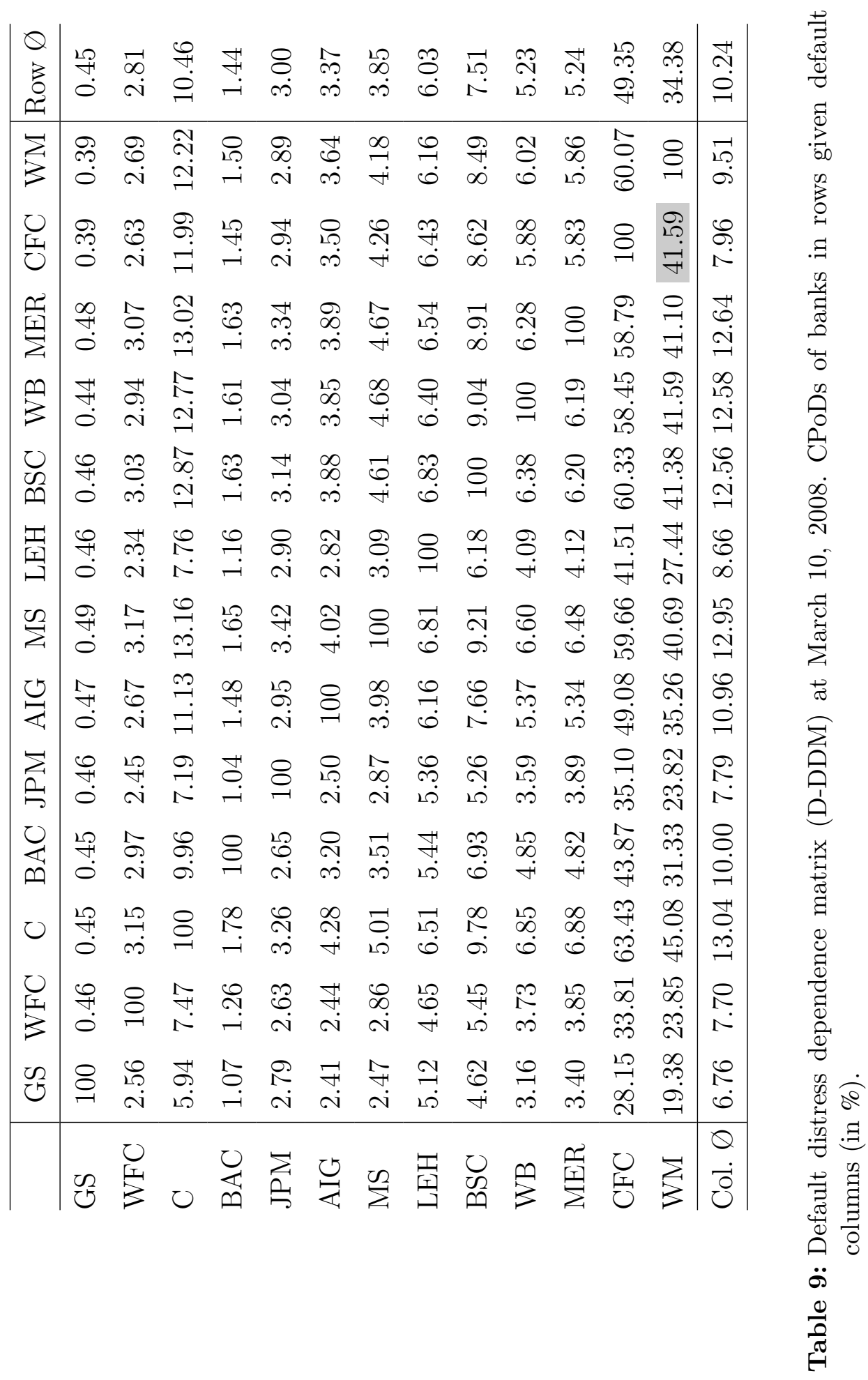



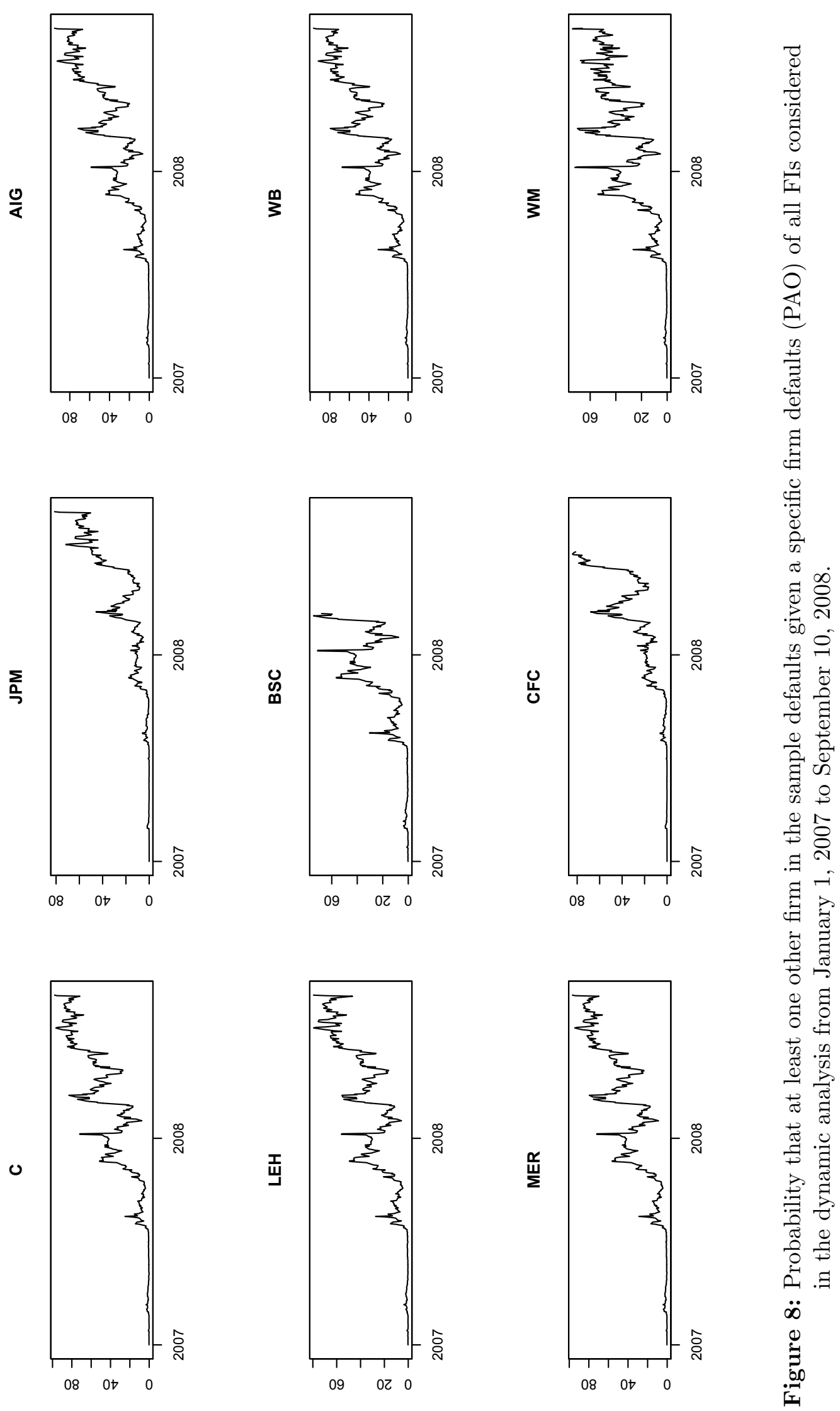

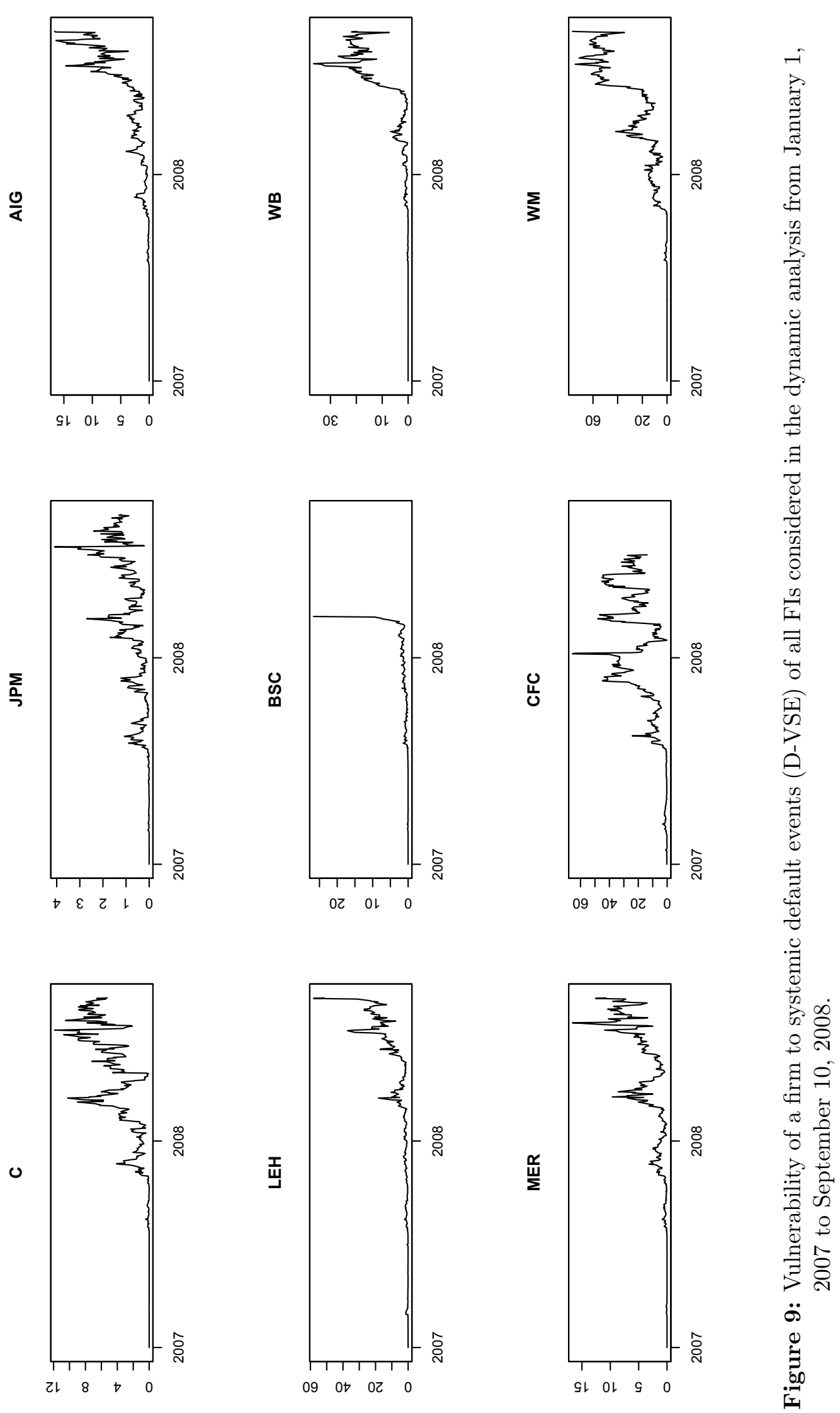

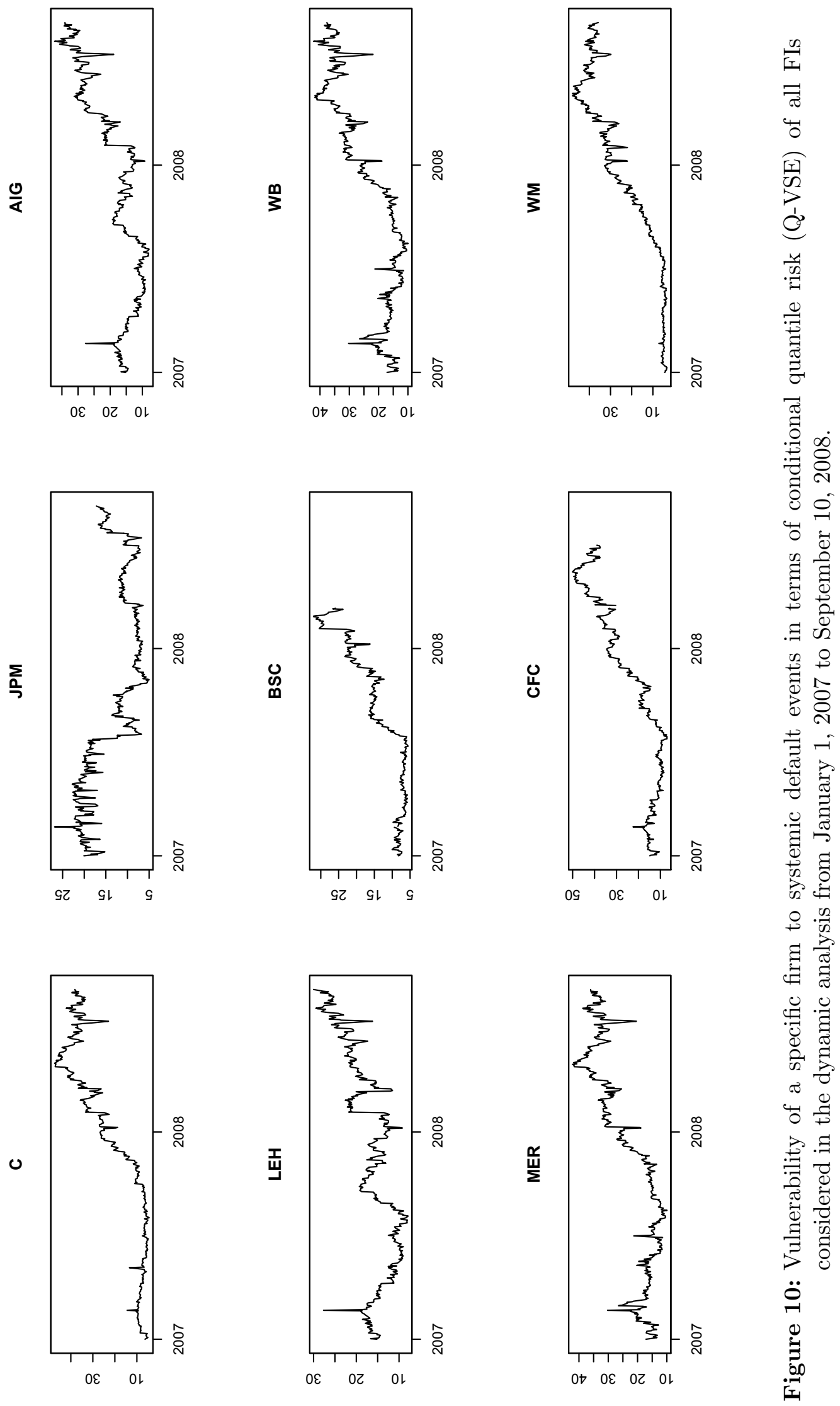